\title{
Identification of fungal fossils and novel azaphilone pigments in ancient carbonised specimens of Hypoxylon fragiforme from forest soils of Châtillon-sur-Seine (Burgundy)
}

\author{
Frank Surup ${ }^{1,2}$ (1) Abolfazl Narmani ${ }^{1,3} \cdot$ Lucile Wendt $^{1,2} \cdot$ Sebastian Pfütze ${ }^{1,2} \cdot$ Robin Kretz $^{1} \cdot$ \\ Kevin Becker ${ }^{1,2} \cdot$ Clément Menbrivès $^{4} \cdot$ Alain Giosa $^{4} \cdot$ Michelle Elliott $^{4} \cdot$ Christophe Petit $^{4}$. \\ Manfred Rohde ${ }^{5} \cdot$ Marc Stadler $^{1,2}$ (D)
}

Received: 7 June 2018/Accepted: 10 September 2018/Published online: 14 September 2018@

\begin{abstract}
Fungal stromata were recently discovered in association with charcoal and burnt soil aggregates during an archaeological survey in the Châtillon-sur-Seine forest massif. The wood and soil in the samples were dated to the medieval period (between 738 and 1411 AD). Light microscopy and scanning electron microscopy revealed that a few of the stromatal fragments still contained ascospores. Their macromorphological characters were described and secondary metabolite profiles were generated using high performance liquid chromatography with diode array and mass spectrometric detection (HPLC-DAD/MS). The combination of these two data lines then allowed species identification. Most of the fragments were assigned to Hypoxylon fragiforme, the type species of the Hypoxylaceae (Xylariales). Two further species whose stromata grew on the fossil charcoal could be tentatively identified as Jackrogersella cohaerens and (more tentatively) as Hypoxylon vogesiacum. These three species are still commonly encountered in the forests of Central Europe today. Furthermore, the HPLC-HRMS data of $H$. fragiforme suggested the presence of unknown azaphilone dimers and of further new pigments. These archaeological compounds were compared to fresh stromata of $H$. fragiforme collected in Germany and subjected to the same analytical protocol. While the major components in both samples were identified as the known mitorubrin type azaphilones and orsellinic acid, the chemical structures of seven novel complex azaphilone pigments, for which we propose the trivial names rutilins C-D and fragirubrins A-E, were elucidated using spectral methods (NMR and CD spectroscopy, high resolution mass spectrometry). It appears that these pigments had indeed persisted for millennia in the fossil stromata.
\end{abstract}

Keywords Biodiversity $\cdot$ Chemotaxonomy $\cdot$ Phylogeny $\cdot$ Sordariomycetes $\cdot$ Xylariales $\cdot$ Structure elucidation

\section{Introduction}

Electronic supplementary material The online version of this article (https://doi.org/10.1007/s13225-018-0412-x) contains supplementary material, which is available to authorized users.

Marc Stadler

marc.stadler@helmholtz-hzi.de

1 Dept. Microbial Drugs, Helmholtz-Zentrum für Infektionsforschung GmbH, Inhoffenstrasse 7, 38124 Braunschweig, Germany

2 German Centre for Infection Research (DZIF), Partner Site Hannover-Braunschweig, 38124 Braunschweig, Germany
The family Hypoxylaceae (Xylariales, Sordariomycetes) is characterised by the presence of manifold stromatal pigments (see recent overview by Helaly et al. 2018), most of

3 Department of Plant Protection, Faculty of Agriculture, University of Tabriz, Tabriz, Iran

4 University of Paris 1 Panthéon-Sorbonne, UMR 7041, Archéologies Environnementales, Paris, France

5 Helmholtz-Zentrum für Infektionsforschung $\mathrm{GmbH}$, Central Facility for Microscopy, Inhoffenstrasse 7, 38124 Braunschweig, Germany 
which belong to the chemical type of azaphilones. In particular the genera Annulohypoxylon, Hypoxylon and Jackrogersella have been thoroughly studied for secondary metabolites, and some of their chemotaxonomic markers have been detected in type specimens and historical herbarium materials originally collected up to over 250 years ago (Stadler et al. 2008a, b). Together with meticulous studies of the morphology of both their asexual and sexual states, these molecular data provided the basis for a taxonomic rearrangement of the major lineages of stromatic Xylariales, resulting in the segregation of the Xylariaceae s. lat. into separate families (Daranagama et al. 2018; Wendt et al. 2018). However, the secondary metabolism of these fungi is so diverse that most compounds produced during their life cycle have not yet been identified. Many metabolites that exhibit prominent biological activities are indeed only produced by the cultures (Surup et al. 2014) or in the growing stromata bearing the anamorph (Stadler et al. 2006), and several were only identified after large-scale biotechnological production and extensive chromatographic work (Kuhnert et al. 2015; Surup et al. 2018).

Other metabolites seem to accumulate in either the stromata or the cultures, and the former compounds in particular can be detected even in ancient specimens.

Recently, some remnants of charred fungal elements were encountered in association with numerous charcoals and burnt soil aggregates during an archaeopedological survey in the Châtillon-sur-Seine forest massif. Macroscopically, these fungi were reminiscent of certain species of the Hypoxylaceae. We chose to study these ancient specimens for the presence of phenotypically informative features, such as the presence of ascospores, by the use of light microscopy, scanning electron microscopy (SEM) and high performance liquid chromatography coupled with diode array detection and mass spectrometry (HPLCDAD/MS) profiling. However, the HPLC profiles displayed peaks that did neither correspond to known secondary metabolites of $H$. fragiforme to any other mitorubrin derivative derived from previous work (e.g. Hellwig et al. 2005; Sir et al. 2016).

In order to find out whether these metabolites constitute artefacts or genuine secondary metabolites that had potentially persisted in the stromata over many centuries, fresh material of the species in question was collected in the field from Germany and their metabolites were isolated to purity and identified by spectral methods. This paper presents the identification the fossil species and the structure elucidation of their secondary metabolites.

\section{Materials and methods}

\section{General}

NMR spectra were recorded with a Bruker Avance III 700 spectrometer with a $5 \mathrm{~mm}$ TCI cryoprobe $\left({ }^{1} \mathrm{H} 700 \mathrm{MHz}\right.$, $\left.{ }^{13} \mathrm{C} 175 \mathrm{MHz}\right)$ and a Bruker Avance III $500\left({ }^{1} \mathrm{H} 500 \mathrm{MHz}\right.$, $\left.{ }^{13} \mathrm{C} 125 \mathrm{MHz}\right)$ spectrometer (Bruker, Bremen, Germany). Chemical shifts $\delta$ were referenced to the solvents chloroform- $d\left({ }^{1} \mathrm{H}, \delta=7.27 \mathrm{ppm} ;{ }^{13} \mathrm{C}, \delta=77.0 \mathrm{ppm}\right)$, acetone- $d_{6}$ $\left({ }^{1} \mathrm{H}, \delta=2.05 \mathrm{ppm} ;{ }^{13} \mathrm{C}, \delta=29.9 \mathrm{ppm}\right)$, pyridine- $d_{5}\left({ }^{1} \mathrm{H}\right.$, $\left.\delta=7.22 \mathrm{ppm} ;{ }^{13} \mathrm{C}, \delta=123.9 \mathrm{ppm}\right)$ and acetonitrile-d $\mathrm{d}_{3}$ $\left({ }^{1} \mathrm{H}, \delta=1.94 \mathrm{ppm} ;{ }^{13} \mathrm{C}, \delta=1.39 \mathrm{ppm}\right)$. Optical rotations were determined using a 241 MC polarimeter (Perkin Elmer, Waltham, USA). UV/Vis spectra were measured with a Shimadzu UV-Vis spectrophotometer UV-2450. CD spectra were recorded with a Jasco J-815 CD spectrometer.

\section{Archaeological material}

The archaeological fungi (Figs. 1, 2 and 3) were collected during an archaeopedological survey of the surroundings of a rural roman settlement known as the "villa de la Pépinière" in the Châtillon-sur-Seine forest massif (Provost 2009). An Airborne Laser Scanner (ALS) using Light Detection and Ranging technique (LiDAR) had indeed revealed a dense network of linear structures all around the archaeological site, which prompted fieldwork to study ancient the agrarian activities of the area, mainly through the analysis of soil characteristics and their biological remains (ecofacts). To do so, the soils of five archaeological contexts were sampled using regular grids of $25 \mathrm{~m}$; 103 pedological pits were described and sampled (10 1 per individualised horizon in each pit). Soil samples were sieved through a $4 \mathrm{~mm}$ and $2 \mathrm{~mm}$ column, and artefacts and ecofacts were recovered, among which a few carbonized fungal elements. The carbonized fungal elements have not been directly dated; however, charcoals and reddish burnt soil recovered in the same sample are dated by radiocarbon and thermoluminescence to the medieval period (between 738 and 1411 AD 2 sigma, 11 dates). As the material is not dated directly and comes from an unstratified context, the fungal fragments could potentially be misdated. We assume nevertheless that the fungi were burnt during the same fire event(s) that produced the charcoal and burnt soil aggregates, and are therefore medieval in date. The rationale for this hypothesis is that the hypoxylaceous fungi, and in particular, Hypoxylon fragiforme, are known to form stromata only on fresh woody substrates in which they even live as endophytes (Chapela et al. 1993; Helaly et al. 2018; Stadler 2011) and 


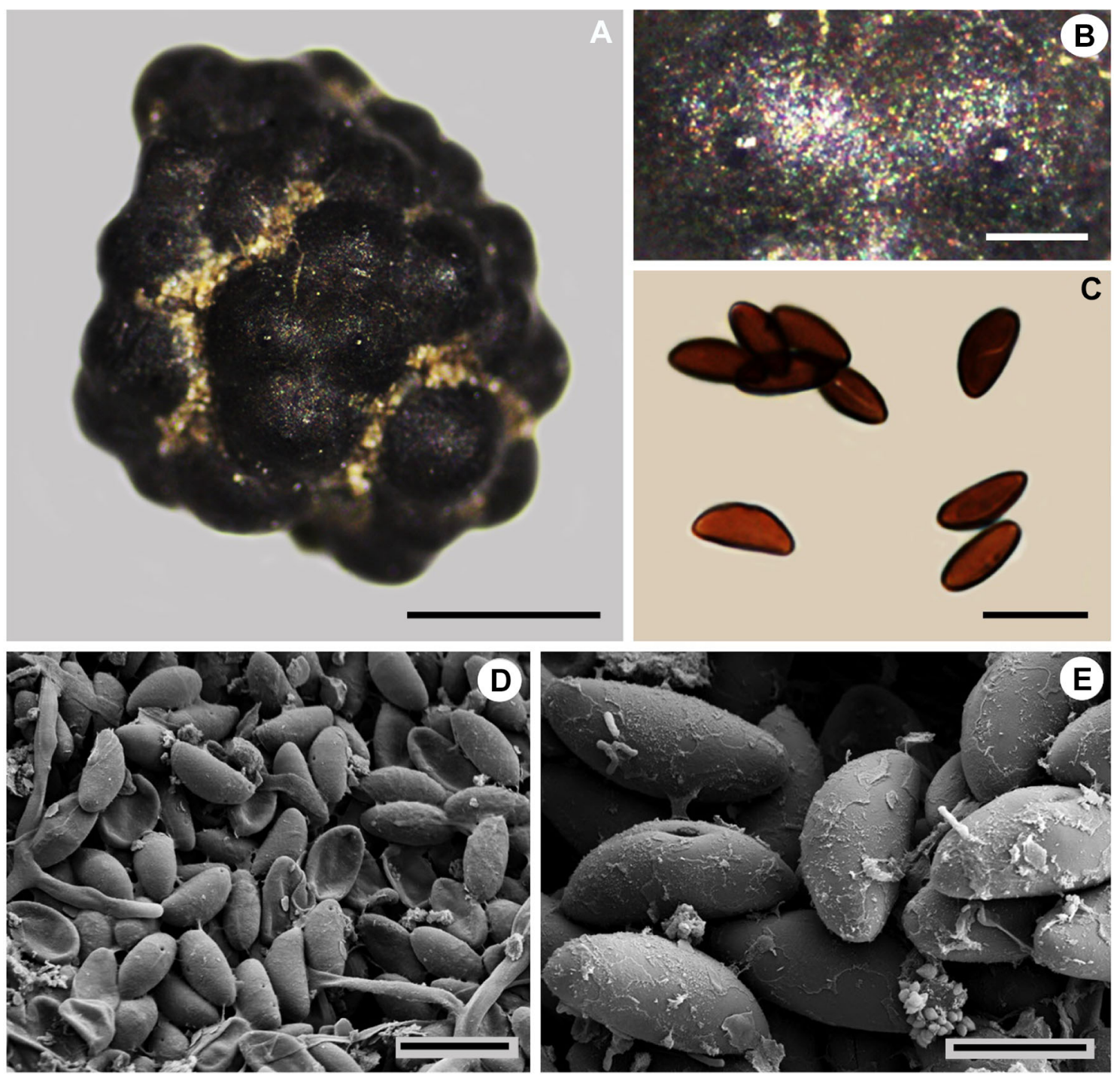

Fig. 1 Hypoxylon fragiforme (Specimen Fo4-2): a Stromatal habit. b Ostioles. c Ascospores by light microscopy. d-e. SEM images of Ascospores. Scale bars: a $1000 \mu \mathrm{m}, \mathbf{b} 200 \mu \mathrm{m}$, c, d $10 \mu \mathrm{m}$, e $5 \mu \mathrm{m}$

could not possibly have grown from the charcoal or the soil.

\section{Microscopy and scanning electron microscopy}

The morphology of macroscopic and microscopic features of the stromata were determined as described by Stadler et al. (2014) and Fournier et al. (2010), also using the key to European species of Hypoxylon s. lat. provided in the latter paper. Photos were taken with a Canon EOS 650D camera through a brightfield microscope at 400-1000 $\times$ magnification or a stereo microscope, respectively. SEM of ascospores were recorded using a field-emission scanning electron microscope (FE-SEM Merlin, Zeiss, Germany), in a similar fashion as reported previously (Sir et al. 2016; Kuhnert et al. 2017).

\section{HPLC profiling}

The stromatal fragments were examined under a dissecting microscope and pieces that obviously contained intact perithecia were singled out and examined using light microscopy. Selected samples that contained ascospores or fragments thereof were chosen for subsequent SEM investigations and also analysed for the presence of secondary metabolites. For this purpose, stromatal extracts were obtained by removing small amounts of the surface and perithecial layer, which were transferred to $1.5 \mathrm{ml}$ reaction tubes. Subsequently, 50-100 $\mu \mathrm{l}$ of acetonitrile were added and the material was extracted for $15 \mathrm{~min}$ at $40{ }^{\circ} \mathrm{C}$ in an ultrasonic bath. The tubes were then centrifuged and the supernatant was used for chemical analyses using high performance liquid chromatography coupled 

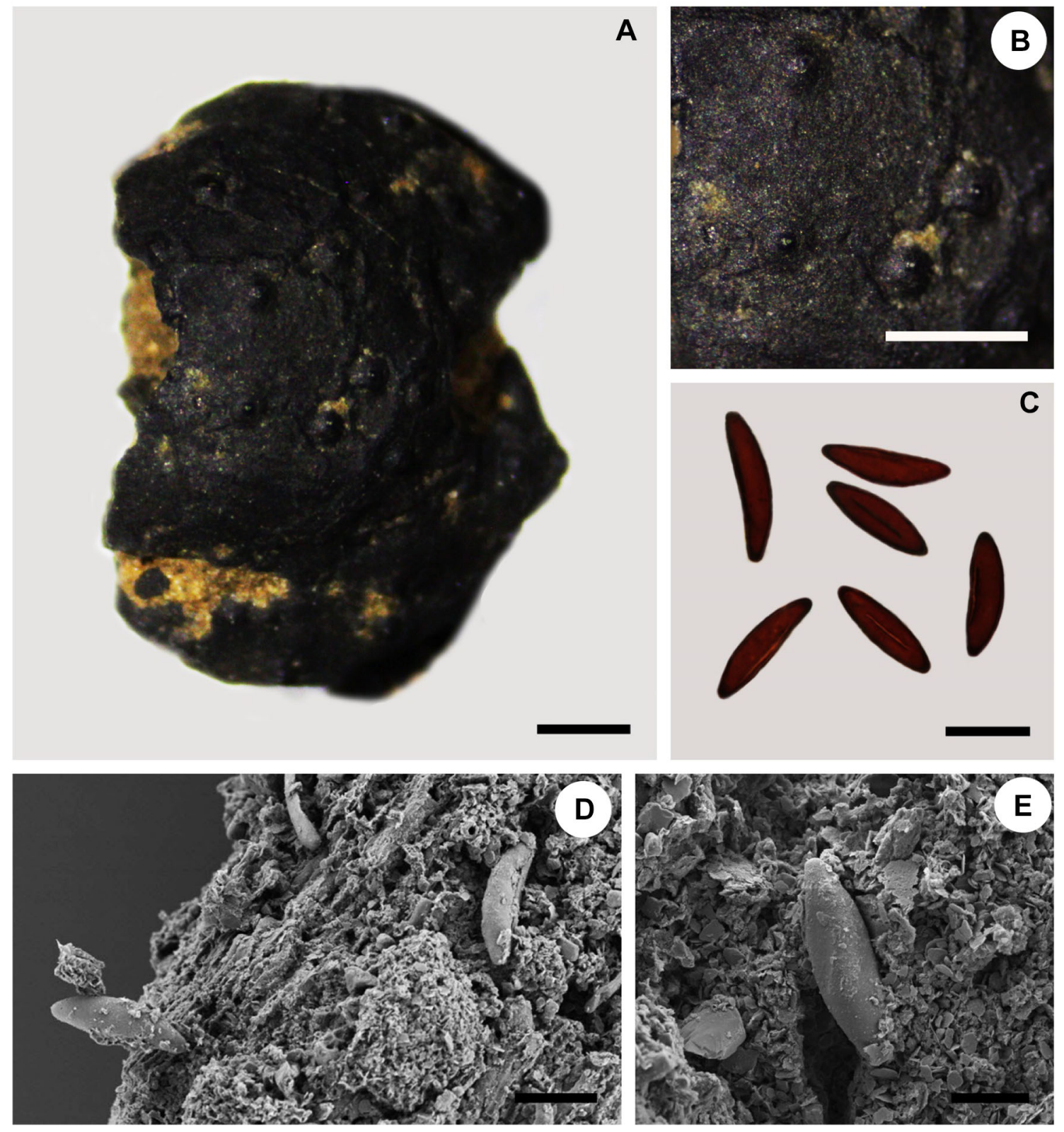

Fig. 2 Hypoxylon cf. vogesiacum (Specimen Fo4-5): a Stromatal fragment. b Ostioles. c Ascospores by light microscopy. d, e SEM images of ascospores. Scale bars: a $1000 \mu \mathrm{m}, \mathbf{b} 200 \mu \mathrm{m}, \mathbf{c}, \mathbf{d} 10 \mu \mathrm{m}$, e $5 \mu \mathrm{m}$

with diode array and electrospray mass spectrometric detection (HPLC/DAD-ESIMS). The instrumental settings and conditions were as described in Kuhnert et al. (2014b). The mass spectra, UV-visible spectra and retention times were compared with an internal database comprising standards of known compounds from Xylariales (cf. Bitzer et al. 2007; Kuhnert et al. 2014a).

\section{Preparation of stromatal extracts for identification of the new pigments and preparative HPLC}

Circa $65 \mathrm{~g}$ of air-dried stromata of Hypoxylon fragiforme were collected by L. Wendt from Fagus sylvatica in the vicinity of Braunschweig, Germany. A voucher specimen, which showed the characteristics of the species, is deposited at the herbarium of M. Stadler (HZI, Braunschweig, Germany; Acc. No. STMA18022). The stromata were extracted with acetone for $3 \times 30 \mathrm{~min}$ in an ultrasonic bath and the extracts were combined and evaporated to dryness 

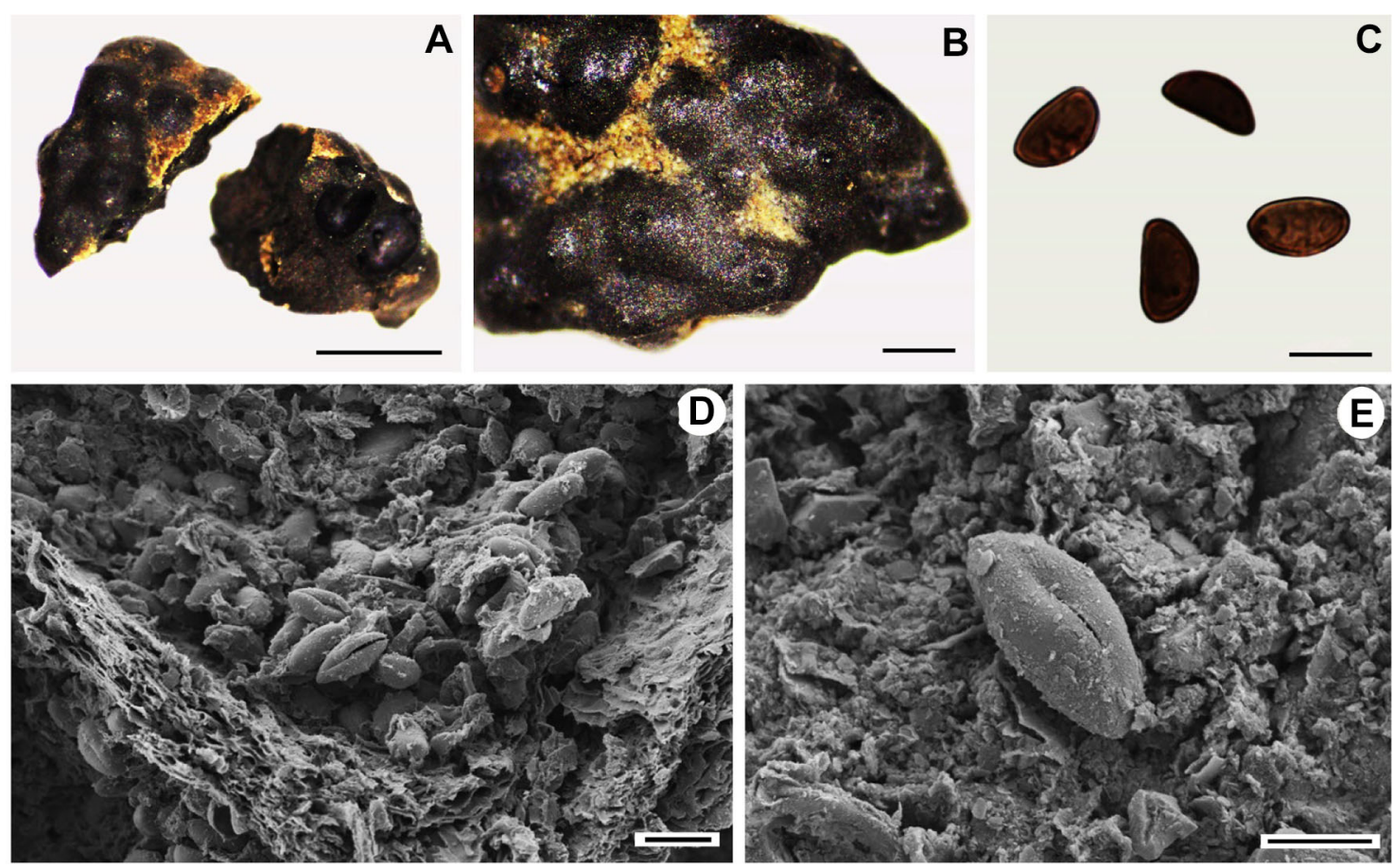

Fig. 3 Jackrogersella cohaerens (Specimen Fo2-1): a Stromata. b Ostioles. c Ascospores by light microscopy. d, e SEM images of ascospores. Scale bars: a $1000 \mu \mathrm{m}, \mathbf{b} 200 \mu \mathrm{m}, \mathbf{c}, \mathbf{d} 10 \mu \mathrm{m}$, e $5 \mu \mathrm{m}$

as described previously (Kuhnert et al. 2015). The crude extract $(6 \mathrm{~g})$ was subjected to normal phase Flash chromatography (GRACE Reveleris ${ }^{\circledR} \mathrm{X} 2$ flash system), using a Reveleris $^{\circledR} 40 \mathrm{~g}$ silica cartridge (particle size: $40 \mu \mathrm{m}$ ). The column was eluted with a ternary gradient mobile phase generated from three solvent mixtures [solvent A: dichloromethane (DCM), solvent B: DCM/acetone (9/1, $\mathrm{v} / \mathrm{v})$, solvent C: acetone] at a flow rate of $40 \mathrm{~mL} / \mathrm{min}$. After isocratic conditions $(100 \% \mathrm{~A})$ for $4 \mathrm{~min}$, the first gradient (AB system) was operating from $0 \% \mathrm{~B}$ to $100 \% \mathrm{~B}$ in 25 min, followed by a second gradient (BC system) from $0 \% \mathrm{C}$ to $100 \% \mathrm{C}$ in $20 \mathrm{~min}$. UV detection was conducted at 220, 254, and $280 \mathrm{~nm}$. Fractions were combined according to the major UV-absorbing peaks to yield 13 main fractions (1-XIII), which were combined and concentrated in vacuo $\left(40{ }^{\circ} \mathrm{C}\right)$ to dryness.

Main fractions obtained this way were further separated via RP-LC with deionized water (solvent $\mathrm{A}$ ) and acetonitrile (B). A Gilson RP-HPLC system (Middleton, Wisconsin, USA) equipped with a GX-271 Liquid handler, a diode array detector (DAD) 172 and a 305 and 306 Pump was utilised to process fractions I, II, and V with a VP Nucleodur C18ec $(150 \times 40 \mathrm{~mm}, 7 \mu \mathrm{m}$; Macherey-Nagel, Düren, Germany) column. The process was performed with a flow rate of $40 \mathrm{ml} / \mathrm{min}$. Prior to the preparative LC experiments, the crude extracts were dissolved in acetonitrile and filtered through a Strata X-33 $\mu \mathrm{m}$ Polymere Reversed Phase Tube (Phenomenex, Aschaffenburg,
Germany) to remove fatty acids and debris. The LC fractions were collected according to the UV absorption at $210 \mathrm{~nm}$. Acetonitrile was evaporated in vacuo, and the aqueous residues were frozen. An Alpha 1-4 LSC freeze dryer (Christ, Osterode, Germany) was used to remove the remaining water.

Fraction V was separated on a gradient from 45 to $80 \%$ solvent B in 40 min with an increase to $100 \%$ B in $5 \mathrm{~min}$, followed by isocratic conditions at $100 \%$ B for 15 min to yield $17.2 \mathrm{mg}$ of $\mathbf{1}$ and $3.89 \mathrm{mg}$ of $\mathbf{8}$. These were eluted as pure compounds after $27.5 \mathrm{~min}$ and $51.5 \mathrm{~min}$, respectively.

Fraction I yielded 3-5 as pure compounds on a gradient starting with isocratic conditions for $5 \mathrm{~min}$ at $60 \% \mathrm{~B}$, followed by a gradient from 60 to $100 \% \mathrm{~B}$ in $45 \mathrm{~min}$, isocratic conditions for $5 \mathrm{~min}$, and a decrease of B to $60 \%$ in $2 \mathrm{~min} .3$ was gained as $37.47 \mathrm{mg}$ with a RT of $48 \mathrm{~min}, \mathbf{4}$ yielded $1.58 \mathrm{mg}$ and eluted after $56 \mathrm{~min}$, while 5 yielded $9.11 \mathrm{mg}$ with a RT of $43 \mathrm{~min}$.

Compounds $6(1.90 \mathrm{mg})$ and $7(3.42 \mathrm{mg})$ were isolated from fraction II on a gradient from 60 to $100 \%$ B in 45 min at RTs of $18.5 \mathrm{~min}$ and $19 \mathrm{~min}$, respectively.

Fraction VI, however, was separated via RP-MPLC (column $480 \times 30 \mathrm{~mm}$, ODS/AQ C18 [Kronlab, Sinsheim, Germany]) with a flow rate of $30 \mathrm{~mL} / \mathrm{min}$ to yield $15.4 \mathrm{mg}$ of 2. It eluted after $56 \mathrm{~min}$ with conditions starting isocratically at $10 \%$ B for $5 \mathrm{~min}$, followed by a gradient to $100 \% \mathrm{~B}$ in $60 \mathrm{~min}$, and isocratic conditions at $100 \% \mathrm{~B}$ for $30 \mathrm{~min}$. 
Rutilin C (1): Red oil; $[\alpha]_{\mathrm{D}}^{25}=+1062^{\circ}\left(c=0.1 \mathrm{CH}_{3} \mathrm{CN}\right)$; $\mathrm{UV}\left(\mathrm{CH}_{3} \mathrm{CN}\right) \lambda_{\max }(\log \varepsilon): 215 \mathrm{~nm}$ (4.61), $266(4.50), 308$ (4.34), 371 (4.60), $442(4.33) \mathrm{nm} ; \mathrm{CD}\left(\mathrm{CH}_{3} \mathrm{CN}\right) \lambda(\Delta \varepsilon)$ : $214(+7.9), 260(-3.5), 312(+7.9), 365(-3.9), 450$ $(+1.3) \mathrm{nm} ;{ }^{1} \mathrm{H}$ NMR $\left(700 \mathrm{MHz}\right.$, acetone- $\left.d_{6}\right): \delta_{\mathrm{H}} 10.78(\mathrm{~s}$, 2'b-OH), 10.72 (s, 2'a-OH), 9.27 (s, 4'b-OH), 9.24 (s, 4' a$\mathrm{OH}), 8.21(\mathrm{~s}, 1 \mathrm{a}-\mathrm{H}), 8.13(\mathrm{dd}, J=15.1 \mathrm{~Hz}, 11.6 \mathrm{~Hz}, 13 \mathrm{a}-$ $\mathrm{H}), 7.64(\mathrm{~d}, J=11.6 \mathrm{~Hz}, 14 \mathrm{a}-\mathrm{H}), 7.04(\mathrm{~d}, J=15.1 \mathrm{~Hz}$, $12 \mathrm{a}-\mathrm{H}), 6.90(\mathrm{~s}, 4 \mathrm{a}-\mathrm{H}), 6.57(\mathrm{dq}, J=15.5 \mathrm{~Hz}, 7.0 \mathrm{~Hz}$, 13b-H), 6.38 (br s, $\left.5^{\prime} \mathrm{b}-\mathrm{H}\right), 6.35$ (br s, $\left.5^{\prime} \mathrm{a}-\mathrm{H}\right), 6.24$ (m, $3^{\prime} \mathrm{b}-$ H), $6.24(\mathrm{~s}, 4 \mathrm{~b}-\mathrm{H}), 6.23\left(\mathrm{~m}, 3^{\prime} \mathrm{a}-\mathrm{H}\right), 6.17$ (dq, $J=15.5 \mathrm{~Hz}$, $1.0 \mathrm{~Hz}, 12 \mathrm{~b}-\mathrm{H}), 5.74(\mathrm{~s}, 6 \mathrm{a}-\mathrm{H}), 5.11(\mathrm{~d}, J=13.5 \mathrm{~Hz}, 1 \mathrm{~b}-$ $\left.\mathrm{H}_{\mathrm{a}}\right), 4.97\left(\mathrm{~d}, J=13.5 \mathrm{~Hz}, 1 \mathrm{~b}-\mathrm{H}_{\mathrm{b}}\right), 2.64\left(\mathrm{~s}, 7^{\prime} \mathrm{b}-\mathrm{H}_{3}\right), 2.60(\mathrm{~s}$, $\left.7^{\prime} \mathrm{a}-\mathrm{H}_{3}\right), 1.90(\mathrm{dd}, J=7.0 \mathrm{~Hz}, 1.0 \mathrm{~Hz}, 14 \mathrm{~b}-\mathrm{H}), 1.73(\mathrm{~s}$, 11b- $\left.\mathrm{H}_{3}\right), 1.66\left(\mathrm{~s}, 11 \mathrm{a}-\mathrm{H}_{3}\right) ;{ }^{13} \mathrm{C}$ NMR $(175 \mathrm{MHz}$, acetone$\left.d_{6}\right): \delta_{\mathrm{C}} 194.1(\mathrm{C}, \mathrm{C}-7 \mathrm{~b}), 192.8$ (C, C-9a), 192.3 (C, C-7a), 188.9 (C, C-9b), 170.7 (C, C-8'a), 170.5 (C, C-8'b), 166.5 (C, C-2'b), 166.2 (C, C-2'a), 164.1 (C, C-4'b), 164.0 (C, C-4'a), 162.5 (C, C-3b), 155.4 (C, C-3a), 155.3 (CH, C1a), 144.94 (C, C-6'b), 144.89 (C, C-6'a), 144.5 (C, C-5b), 143.0 (C, C-5a), 140.8 (CH, C-14a), 135.9 (CH, C-13b), $135.6(\mathrm{CH}, \mathrm{C}-12 \mathrm{a}), 131.5(\mathrm{CH}, \mathrm{C}-13 \mathrm{a}), 129.2(\mathrm{C}, \mathrm{C}-6 \mathrm{~b})$, 125.9 (CH, C-12b), 116.1 (C, C-10b), 115.9 (CH, C-4a), $115.6(\mathrm{C}, \mathrm{C}-10 \mathrm{a}), 112.8\left(\mathrm{CH}, \mathrm{C}-5^{\prime} \mathrm{b}\right), 112.7\left(\mathrm{CH}, \mathrm{C}-5^{\prime} \mathrm{a}\right)$, $110.0(\mathrm{CH}, \mathrm{C}-6 \mathrm{a}), 105.0$ (C, C-1'a), $104.8\left(\mathrm{C}, \mathrm{C}-1^{\prime} \mathrm{b}\right)$, $101.81\left(\mathrm{CH}, \mathrm{C}-3^{\prime} \mathrm{b}\right), 101.76\left(\mathrm{CH}, \mathrm{C}-3^{\prime} \mathrm{a}\right), 98.5$ (CH, C-4b), 86.82 (C, C-8b), 86.75 (C, C-8a), $64.8\left(\mathrm{CH}_{2}, \mathrm{C}-1 \mathrm{~b}\right), 24.3$ $\left(\mathrm{CH}_{3}, \mathrm{C}-7^{\prime} \mathrm{b}\right), 24.1\left(\mathrm{CH}_{3}, \mathrm{C}-7^{\prime} \mathrm{a}\right), 22.73\left(\mathrm{CH}_{3}, \mathrm{C}-11 \mathrm{~b}\right)$, $22.69\left(\mathrm{CH}_{3}, \mathrm{C}-11 \mathrm{a}\right), 18.9\left(\mathrm{CH}_{3}, \mathrm{C}-14 \mathrm{~b}\right) ;$ ESIMS $\mathrm{m} / \mathrm{z}$ 763.22 $[\mathrm{M}+\mathrm{H}]^{+}, 761.22[\mathrm{M}-\mathrm{H}]^{-}$; HRESIMS $\mathrm{m} / \mathrm{z}$ 763.2014 $[\mathrm{M}+\mathrm{H}]^{+}$(calcd. for $\mathrm{C}_{42} \mathrm{H}_{35} \mathrm{O}_{14}, 763.2012$ ); $\mathrm{R}_{\mathrm{t}}=13.5 \mathrm{~min}$.

Rutilin D (2): Yellow oil; $[\alpha]_{\mathrm{D}}^{25}=+6^{\circ}\left(c=0.1 \mathrm{CH}_{3} \mathrm{CN}\right)$; $\mathrm{UV}\left(\mathrm{CH}_{3} \mathrm{CN}\right) \lambda_{\max }(\log \varepsilon): 215$ (3.66), 266 (3.45), 310 (3.29), 366 (3.40), $441(3.13) \mathrm{nm} ; \mathrm{CD}\left(\mathrm{CH}_{3} \mathrm{CN}\right) \lambda(\Delta \varepsilon)$ : $262(-3.7), 319(+11.2), 366(-0.4), 416(+2.9) \mathrm{nm} ;{ }^{1} \mathrm{H}$ NMR (700 MHz, $\left.\mathrm{CH}_{3} \mathrm{CN}-d_{3}\right): \delta_{\mathrm{H}} 10.59\left(\mathrm{~s}, 2^{\prime} \mathrm{b}-\mathrm{OH}\right), 10.54$ (s, 2'a-OH), $8.04(\mathrm{~s}, 1 \mathrm{a}-\mathrm{H}), 8.04(\mathrm{~m}, 13 \mathrm{a}-\mathrm{H}), 7.42(\mathrm{~d}$, $J=11.8 \mathrm{~Hz}, 14 \mathrm{a}-\mathrm{H}), 6.88(\mathrm{~d}, J=15.1 \mathrm{~Hz}, 12 \mathrm{a}-\mathrm{H}), 6.74$ (s, 4a-H), 6.51 (dd, $J=15.6 \mathrm{~Hz}, 5.5 \mathrm{~Hz}, 13 \mathrm{~b}-\mathrm{H}), 6.35$ (m, 12b-H), 6.32 (m, 5'a-H, 5'b-H), 6.23 (d, $J=2.6 \mathrm{~Hz}, 3^{\prime} \mathrm{b}-$ H), $6.21\left(\mathrm{~d}, J=2.6 \mathrm{~Hz}, 3^{\prime} \mathrm{a}-\mathrm{H}\right), 6.19(\mathrm{~s}, 4 \mathrm{~b}-\mathrm{H}), 5.70(\mathrm{~d}$, $J=0.9 \mathrm{~Hz}, 6 \mathrm{a}-\mathrm{H}), 5.11\left(\mathrm{br} \mathrm{d}, J=14.0 \mathrm{~Hz}, 1 \mathrm{~b}-\mathrm{H}_{\mathrm{a}}\right), 4.99$ (br d, $J=14.0 \mathrm{~Hz}, 1 \mathrm{~b}-\mathrm{H}_{\mathrm{b}}$ ), $4.74(\mathrm{br} \mathrm{d}, J=5.5 \mathrm{~Hz}, 14 \mathrm{~b}-$ $\left.\mathrm{H}_{2}\right), 2.60\left(\mathrm{~s}, 7^{\circ} \mathrm{b}-\mathrm{H}_{3}\right), 2.57\left(\mathrm{~s}, 7^{\prime} \mathrm{a}-\mathrm{H}_{3}\right), 2.09$ (s, 16b- $\left.\mathrm{H}_{3}\right)$, $1.68\left(\mathrm{~s}, 11 \mathrm{~b}-\mathrm{H}_{3}\right), 1.64\left(\mathrm{~s}, 11 \mathrm{a}-\mathrm{H}_{3}\right) ;{ }^{13} \mathrm{C}$ NMR $(175 \mathrm{MHz}$, $\left.\mathrm{CH}_{3} \mathrm{CN}-d_{3}\right): \delta_{\mathrm{C}} 194.4(\mathrm{C}, \mathrm{C}-7 \mathrm{~b}), 193.3(\mathrm{C}, \mathrm{C}-9 \mathrm{a}), 192.9$ (C, C-7a), 189.4 (C, C-9b), 171.4 (C, C-15b), 174.6 (C, C-8'a, C-8'b), 166.2 (C, C-2'b), 165.9 (C, C-2'a), 163.8 (C, C-4'a), 163.7 (C, C-4'b), 161.4 (C, C-3b), 155.6 (CH, C-1a), 155.5 (C, C-3a), 145.31 (C, C-6'b), 145.25 (C, C6'a), 144.3 (C, C-5b), 143.7 (C, C-5a), 141.2 (CH, C-14a),
135.6 (CH, C-12a), 133.2 (CH, C-13b), 131.4 (CH, C13a), 129.1 (C, C-6b), 125.6 (CH, C-12b), 116.9 (C, C10b), 116.1 (CH, C-4a), 115.6 (C, C-10a), 112.84 (CH, C5'b), $112.75\left(\mathrm{CH}, \mathrm{C}-5^{\prime} \mathrm{a}\right), 109.7$ (CH, C-6a), $105.2(\mathrm{C}, \mathrm{C}-$ $\left.1^{\prime} \mathrm{a}\right), 105.0\left(\mathrm{C}, \mathrm{C}-1^{\prime} \mathrm{b}\right), 101.83\left(\mathrm{CH}, \mathrm{C}-3^{\prime} \mathrm{a}\right), 101.80(\mathrm{CH}$, $\left.\mathrm{C}-3^{\prime} \mathrm{b}\right), 100.7(\mathrm{CH}, \mathrm{C}-4 \mathrm{~b}), 87.2$ (C, C-8a, C-8b), 65.0 $\left(\mathrm{CH}_{2}, \mathrm{C}-1 \mathrm{~b}\right), 64.2\left(\mathrm{CH}_{2}, \mathrm{C}-14 \mathrm{~b}\right), 24.2\left(\mathrm{CH}_{3}, \mathrm{C}-7{ }^{`} \mathrm{~b}\right), 24.0$ $\left(\mathrm{CH}_{3}, \mathrm{C}-7^{\prime} \mathrm{a}\right), 22.9\left(\mathrm{CH}_{3}, \mathrm{C}-11 \mathrm{a}\right), 22.8\left(\mathrm{CH}_{3}, \mathrm{C}-11 \mathrm{~b}\right), 21.1$ $\left(\mathrm{CH}_{3}, \mathrm{C}-16 \mathrm{~b}\right)$; ESIMS $\mathrm{m} / \mathrm{z}, 763.22[\mathrm{M}+\mathrm{H}]^{+}, 761.22$ $[\mathrm{M}-\mathrm{H}]^{-}$; HRESIMS $m / z$ 821.2063 $[\mathrm{M}+\mathrm{H}]^{+}$(calcd. for $\left.\mathrm{C}_{44} \mathrm{H}_{37} \mathrm{O}_{16}, 821.2076\right) ; \mathrm{R}_{\mathrm{t}}=12.7 \mathrm{~min}$.

Fragirubrin A (3): Yellow oil; $[\alpha]_{\mathrm{D}}^{25}=+447^{\circ}(c=0.1$ $\left.\mathrm{CH}_{3} \mathrm{CN}\right)$; $\mathrm{UV}\left(\mathrm{CH}_{3} \mathrm{CN}\right) \lambda_{\max }(\log \varepsilon): 218$ (4.27), 326 (4.26) $\mathrm{nm} ; \mathrm{CD}\left(\mathrm{CH}_{3} \mathrm{CN}\right) \lambda(\Delta \varepsilon): 199(-7.1), 231(+1.8), 248$ $(-1,3), 273(+4.8), 322(-4.9) \mathrm{nm} ;{ }^{1} \mathrm{H}$ NMR $(700 \mathrm{MHz}$, $\left.\mathrm{CHCl}_{3}-d\right): \delta_{\mathrm{H}} 7.85(\mathrm{~d}, J=0.9 \mathrm{~Hz}, 1-\mathrm{H}), 6.14(\mathrm{~s}, 4-\mathrm{H})$, $5.54(\mathrm{~d}, J=0.9 \mathrm{~Hz}, 5-\mathrm{H}), 5.17(\mathrm{dqd}, J=7.4,6.5,5.3 \mathrm{~Hz}$, $11-\mathrm{H}), 2.69\left(\mathrm{dd}, J=15.0,7.4 \mathrm{~Hz}, 10-\mathrm{H}_{\mathrm{a}}\right), 2.61(\mathrm{dd}$, $\left.J=15.0,5.3 \mathrm{~Hz}, 10-\mathrm{H}_{\mathrm{b}}\right), 2.45\left(\mathrm{t}, J=7.5 \mathrm{~Hz}, 16-\mathrm{H}_{2}\right), 1.63$ $\left(\mathrm{m}, 17-\mathrm{H}_{2}\right), 2.45\left(\mathrm{t}, J=7.5 \mathrm{~Hz}, 16-\mathrm{H}_{2}\right), 2.06\left(\mathrm{~s}, 14-\mathrm{H}_{3}\right)$, $1.53\left(\mathrm{~s}, 9-\mathrm{H}_{3}\right), 1.33\left(\mathrm{~d}, J=6.5 \mathrm{~Hz}, 12-\mathrm{H}_{3}\right), 1.33(\mathrm{~m}, 18$ $\left.\mathrm{H}_{2}\right), 1.24-1.31\left(\mathrm{~m}, 19-\mathrm{H}_{2}-29-\mathrm{H}_{2}\right), 0.89$ (t, $J=7.1 \mathrm{~Hz}, 30$ $\left.\mathrm{H}_{3}\right) ;{ }^{13} \mathrm{C}$ NMR (175 MHz, $\left.\mathrm{CHCl}_{3}-d\right): \delta_{\mathrm{C}} 193.2(\mathrm{C}, \mathrm{C}-8)$, 192.8 (C, C-6), 173.1 (C, C-15), 170.3 (C, C-13), 157.8 (C, C-3), 153.7 (CH, C-1), 141.9 (C, C-4a), 115.2 (C, C8a), 110.8 (CH, C-4), 107.5 (CH, C-5), 84.1 (C, C-7), 67.5 (CH, C-11), $39.4\left(\mathrm{CH}_{2}, \mathrm{C}-10\right), 33.2\left(\mathrm{CH}_{2}, \mathrm{C}-16\right), 31.9$ $\left(\mathrm{CH}_{2}, \mathrm{C}-28\right), 29.68,29.67,29.66,29.64,29.63,29.61$, 29.41, 29.35, $29.26\left(9 \times \mathrm{CH}_{2}, \mathrm{C}-19-\mathrm{C}-27\right), 28.9\left(\mathrm{CH}_{2}, \mathrm{C}-\right.$ 18), $24.6\left(\mathrm{CH}_{2}, \mathrm{C}-17\right), 22.7\left(\mathrm{CH}_{2}, \mathrm{C}-29\right), 22.1\left(\mathrm{CH}_{3}, \mathrm{C}-9\right)$, $21.2\left(\mathrm{CH}_{2}, \mathrm{C}-14\right), 19.8\left(\mathrm{CH}_{3}, \mathrm{C}-12\right), 14.1\left(\mathrm{CH}_{3}, \mathrm{C}-30\right)$; ESIMS $m / z 531.32\left[\mathrm{M}+\mathrm{H}^{+}, 529.27[\mathrm{M}-\mathrm{H}]^{-}\right.$; HRESIMS $\mathrm{m} / \mathrm{z} 531.3316[\mathrm{M}+\mathrm{H}]^{+}$(calcd. for $\mathrm{C}_{31} \mathrm{H}_{47} \mathrm{O}_{7}$, 531.3316); $\mathrm{R}_{\mathrm{t}}=18.8$ min.

Fragirubrin B (4): Yellow oil; $[\alpha]_{\mathrm{D}}^{25}=-86^{\circ}(c=0.1$ $\mathrm{CH}_{3} \mathrm{CN}$ ); UV $\left(\mathrm{CH}_{3} \mathrm{CN}\right) \lambda_{\max }(\log \varepsilon): 219$ (4.16), $327 \mathrm{~nm}$ (4.16); $\mathrm{CD}\left(\mathrm{CH}_{3} \mathrm{CN}\right) \lambda(\Delta \varepsilon): 199(-1.8), 232(+0.7), 249(-$ $0.4), 274(+1.6), 323(-1.4) \mathrm{nm} ;{ }^{1} \mathrm{H}$ NMR $(700 \mathrm{MHz}$, $\left.\mathrm{CHCl}_{3}-d\right): \delta_{\mathrm{H}} 7.85(\mathrm{br} \mathrm{s}, 1-\mathrm{H}), 6.14(\mathrm{~s}, 4-\mathrm{H}), 5.54$ (br s, 5H), $5.17(\mathrm{dqd}, J=7.4,6.5,5.3 \mathrm{~Hz}, 11-\mathrm{H}), 2.69(\mathrm{dd}$, $\left.J=15.0,7.4 \mathrm{~Hz}, 10-\mathrm{H}_{\mathrm{a}}\right), 2.61(\mathrm{dd}, J=15.0,5.3 \mathrm{~Hz}, 10$ $\left.\mathrm{H}_{\mathrm{b}}\right), 2.45\left(\mathrm{t}, J=7.5 \mathrm{~Hz}, 16-\mathrm{H}_{2}\right), 2.06\left(\mathrm{~s}, 14-\mathrm{H}_{3}\right), 1.63(\mathrm{~m}$, $\left.17-\mathrm{H}_{2}\right), 1.53\left(\mathrm{~s}, 9-\mathrm{H}_{3}\right), 1.33\left(\mathrm{~d}, J=6.5 \mathrm{~Hz}, 12-\mathrm{H}_{3}\right), 1.33$ $\left(\mathrm{m}, \quad 18-\mathrm{H}_{2}\right), \quad 1.24-1.31\left(\mathrm{~m}, 19-\mathrm{H}_{2}-31-\mathrm{H}_{2}\right), 0.89 \quad(\mathrm{t}$, $\left.J=7.0 \mathrm{~Hz}, 32-\mathrm{H}_{3}\right) ;{ }^{13} \mathrm{C}$ NMR $\left(175 \mathrm{MHz}, \mathrm{CHCl}_{3}-d\right): \delta_{\mathrm{C}}$ 193.2 (C, C-8), 192.8 (C, C-6), 173.1 (C, C-15), 170.2 (C, C-13), 157.8 (C, C-3), 153.7 (CH, C-1), 141.9 (C, C-4a), 115.2 (C, C-8a), 110.8 (CH, C-4), $107.5(\mathrm{CH}, \mathrm{C}-5), 84.1$ $(\mathrm{C}, \mathrm{C}-7), 67.5(\mathrm{CH}, \mathrm{C}-11), 39.4\left(\mathrm{CH}_{2}, \mathrm{C}-10\right), 33.2\left(\mathrm{CH}_{2}\right.$, $\mathrm{C}-16), 31.9\left(\mathrm{CH}_{2}, \mathrm{C}-30\right), 29.68,29.67,29.66,29.64$, $29.63,29.61,29.42,29.35,29.26\left(11 \times \mathrm{CH}_{2}, \mathrm{C}-19-\mathrm{C}-\right.$ 29), $28.9\left(\mathrm{CH}_{2}, \mathrm{C}-18\right), 24.6\left(\mathrm{CH}_{2}, \mathrm{C}-17\right), 22.7\left(\mathrm{CH}_{2}, \mathrm{C}-\right.$ 
31), $22.1\left(\mathrm{CH}_{3}, \mathrm{C}-9\right), 21.2\left(\mathrm{CH}_{2}, \mathrm{C}-14\right), 19.8\left(\mathrm{CH}_{3}, \mathrm{C}-12\right)$, $14.1\left(\mathrm{CH}_{3}, \mathrm{C}-32\right)$; ESIMS $\mathrm{m} / z 559.42[\mathrm{M}+\mathrm{H}]^{+}, 557.32$ $[\mathrm{M}-\mathrm{H}]^{-}$; HRESIMS $m / z 559.3635[\mathrm{M}+\mathrm{H}]^{+}$(calcd. for $\left.\mathrm{C}_{33} \mathrm{H}_{51} \mathrm{O}_{7}, 559.3629\right) ; \mathrm{R}_{\mathrm{t}}=19.8 \mathrm{~min}$.

Fragirubrin $\mathrm{C}(\mathbf{5})$ : Yellow oil; $[\alpha]_{\mathrm{D}}^{25}=+1206^{\circ}(c=1.0$ $\left.\mathrm{CH}_{3} \mathrm{CN}\right)$; UV $\left(\mathrm{CH}_{3} \mathrm{CN}\right) \lambda_{\max }(\log \varepsilon): 219(4.34), 327 \mathrm{~nm}$ (4.34); $\mathrm{CD}\left(\mathrm{CH}_{3} \mathrm{CN}\right) \lambda(\Delta \varepsilon): 201(-6.2), 232(+1.6), 249$ $(-1.1), 272(+4.6), 322(-4.6) \mathrm{nm} ;{ }^{1} \mathrm{H}$ NMR $(700 \mathrm{MHz}$, CHCl- $\left.d_{6}\right): \delta_{\mathrm{H}} 7.85(\mathrm{br} \mathrm{s}, 1-\mathrm{H}), 6.14(\mathrm{~s}, 4-\mathrm{H}), 5.54$ (br s, 5H), 5.30-5.41 (m, 23-H, 24-H, 26-H, 27-H), 5.17 (dqd, $J=7.4,6.5,5.3 \mathrm{~Hz}, 11-\mathrm{H}), 2.77\left(\mathrm{t}, J=7.0 \mathrm{~Hz}, 25-\mathrm{H}_{2}\right)$, $2.69\left(\mathrm{dd}, J=15.0,7.4 \mathrm{~Hz}, 10-\mathrm{H}_{\mathrm{a}}\right), 2.61(\mathrm{dd}, J=15.0$, $\left.5.3 \mathrm{~Hz}, 10-\mathrm{H}_{\mathrm{b}}\right), 2.44\left(\mathrm{t}, J=7.5 \mathrm{~Hz}, 16-\mathrm{H}_{2}\right), 2.05(\mathrm{~s}, 14-$ $\left.\mathrm{H}_{3}\right), 2.07-2.02\left(\mathrm{~s}, 22-\mathrm{H}_{2}, 28-\mathrm{H}_{2}\right), 1.63\left(\mathrm{~m}, 17-\mathrm{H}_{2}\right), 1.53(\mathrm{~s}$, 9- $\left.\mathrm{H}_{3}\right), 1.32\left(\mathrm{~d}, J=6.2 \mathrm{~Hz}, 12-\mathrm{H}_{3}\right), 1.24-1.38\left(\mathrm{~m}, 18-\mathrm{H}_{2}-\right.$ $\left.21-\mathrm{H}_{2}, 28-\mathrm{H}_{2}-31-\mathrm{H}_{2}\right), 0.89\left(\mathrm{t}, J=6.8 \mathrm{~Hz}, 32-\mathrm{H}_{3}\right) ;{ }^{13} \mathrm{C}$ NMR (175 MHz, $\left.\mathrm{CHCl}_{3}-d\right): \delta_{\mathrm{C}} 193.2(\mathrm{C}, \mathrm{C}-8), 192.8(\mathrm{C}$, C-6), 173.1 (C, C-15), 170.2 (C, C-13), 157.8 (C, C-3), 153.7 (CH, C-1), 141.9 (C, C-4a), $130.2(\mathrm{CH}, \mathrm{CH}-23)^{* 1}$, $130.1(\mathrm{CH}, \mathrm{C}-27)^{* 1}, 127.94(\mathrm{C}, \mathrm{CH}-24)^{* 2}, 127.92(\mathrm{CH}$, $\mathrm{C}-26)^{2}, 115.2(\mathrm{C}, \mathrm{C}-8 \mathrm{a}), 110.8(\mathrm{CH}, \mathrm{C}-4), 107.5(\mathrm{CH}, \mathrm{C}-$ 5), $84.1(\mathrm{C}, \mathrm{C}-7), 67.5(\mathrm{CH}, \mathrm{C}-11), 39.4\left(\mathrm{CH}_{2}, \mathrm{C}-10\right), 33.2$ $\left(\mathrm{CH}_{2}, \mathrm{C}-16\right), 31.49\left(\mathrm{CH}_{2}, \mathrm{C}-28\right)^{* 3}, 31.48\left(\mathrm{CH}_{2}, \mathrm{C}-30\right)^{* 3}$, 29.6, 29.3, 29.14, 29.06, $28.9\left(5 \times \mathrm{CH}_{2}, \mathrm{C}-18-\mathrm{C}-21, \mathrm{C}-\right.$ 29), $27.18\left(\mathrm{CH}_{2}, \mathrm{C}-22\right)^{* 4}, 27.16\left(\mathrm{CH}_{2}, \mathrm{C}-28\right)^{* 4}, 25.6$ $\left(\mathrm{CH}_{2}, \mathrm{C}-25\right), 24.6\left(\mathrm{CH}_{2}, \mathrm{C}-17\right), 22.5\left(\mathrm{CH}_{3}, \mathrm{C}-31\right), 22.1$ $\left(\mathrm{CH}_{3}, \mathrm{C}-9\right), 21.2\left(\mathrm{CH}_{3}, \mathrm{C}-14\right), 19.8\left(\mathrm{CH}_{3}, \mathrm{C}-12\right), 14.1$ $\left(\mathrm{CH}_{3}, \mathrm{C}-31\right), *^{1}, *^{2}, *^{3}, *^{4}$ assignments might be interchanged; ESIMS $\mathrm{m} / \mathrm{z} 555.39[\mathrm{M}+\mathrm{H}]^{+}$; HRESIMS $\mathrm{m} / \mathrm{z}$ 555.3322 $[\mathrm{M}+\mathrm{H}]^{+}$(calcd. for $\mathrm{C}_{33} \mathrm{H}_{47} \mathrm{O}_{7}, 555.3316$ ); $\mathrm{R}_{\mathrm{t}}=18.1 \mathrm{~min}$.

Fragirubrin D (6): Yellow oil; $[\alpha]_{\mathrm{D}}^{25}=+56^{\circ} \quad(c=1.0$ $\left.\mathrm{CH}_{3} \mathrm{CN}\right) ; \mathrm{UV}\left(\mathrm{CH}_{3} \mathrm{CN}\right) \lambda_{\max }(\log \varepsilon): 221$ (4.11), 327 (4.08) $\mathrm{nm} ; \mathrm{CD}\left(\mathrm{CH}_{3} \mathrm{CN}\right) \lambda(\Delta \varepsilon): 202(-2.3), 230(+1.1), 248$ $(-0.3), 273(+2.0), 323(-1.9) \mathrm{nm} ;{ }^{1} \mathrm{H}$ NMR $(700 \mathrm{MHz}$, $\left.\mathrm{CHCl}_{3}-d\right): \delta_{\mathrm{H}} 7.86(\mathrm{~d}, J=0.9 \mathrm{~Hz}, 1-\mathrm{H}), 6.14(\mathrm{~s}, 4-\mathrm{H})$, $5.54(\mathrm{~d}, J=0.9 \mathrm{~Hz}, 5-\mathrm{H}), 5.30-5.41$ (m, 23-H, 24-H, 26$\mathrm{H}, 27-\mathrm{H}), 5.17$ (dqd, $J=7.4,6.5,5.3 \mathrm{~Hz}, 11-\mathrm{H}), 3.81(\mathrm{~m}$, 29-H), $2.78\left(\mathrm{t}, J=6.2 \mathrm{~Hz}, 25-\mathrm{H}_{2}\right), 2.69$ (dd, $J=14.8$, $\left.7.5 \mathrm{~Hz}, 10-\mathrm{H}_{\mathrm{a}}\right), 2.61\left(\mathrm{dd}, J=14.8,5.4 \mathrm{~Hz}, 10-\mathrm{H}_{\mathrm{b}}\right), 2.45(\mathrm{t}$, $\left.J=7.4 \mathrm{~Hz}, 16-\mathrm{H}_{2}\right), 2.09\left(\mathrm{~m}, 28-\mathrm{H}_{2}\right), 2.06\left(\mathrm{~s}, 14-\mathrm{H}_{3}\right), 2.03$ $\left(\mathrm{s}, 22-\mathrm{H}_{2}\right), 1.63\left(\mathrm{~m}, 17-\mathrm{H}_{2}\right), 1.53\left(\mathrm{~s}, 9-\mathrm{H}_{3}\right), 1.48(\mathrm{~m}, 29-$ $\left.\mathrm{H}_{\mathrm{a}}, 30-\mathrm{H}_{2}\right), 1.41\left(\mathrm{~m}, 29-\mathrm{H}_{\mathrm{b}},\right), 1.33\left(\mathrm{~d}, J=6.2 \mathrm{~Hz}, 12-\mathrm{H}_{3}\right)$, $1.24-1.38\left(\mathrm{~m}, 18-\mathrm{H}_{2}-21-\mathrm{H}_{2}\right), 1.20(\mathrm{~d}, J=6.5 \mathrm{~Hz}, 32-$ $\left.\mathrm{H}_{3}\right) ;{ }^{13} \mathrm{C}$ NMR $\left(175 \mathrm{MHz}, \mathrm{CHCl}_{3}-d\right): \delta_{\mathrm{C}} 193.2(\mathrm{C}, \mathrm{C}-8)$, 192.9 (C, C-6), 173.1 (C, C-15), 170.3 (C, C-13), 157.8 (C, C-3), 153.7 (CH, C-1), 142.0 (C, C-4a), $130.2(\mathrm{CH}$, $\mathrm{CH}-23)^{* 1}, 129.6(\mathrm{CH}, \mathrm{C}-27)^{* 1}, 128.4(\mathrm{C}, \mathrm{CH}-24)^{* 2}$, $127.8(\mathrm{CH}, \mathrm{C}-26)^{* 2}, 115.2(\mathrm{C}, \mathrm{C}-8 \mathrm{a}), 110.8(\mathrm{CH}, \mathrm{C}-4)$, $107.5(\mathrm{CH}, \mathrm{C}-5), 84.1$ (C, C-7), $68.5(\mathrm{CH}, \mathrm{C}-31), 67.5$ (CH, C-11), $39.4\left(\mathrm{CH}_{2}, \mathrm{C}-10\right), 38.9\left(\mathrm{CH}_{2}, \mathrm{C}-30\right), 33.2$ $\left(\mathrm{CH}_{2}, \mathrm{C}-16\right), 29.6,29.3,29.14,29.06,28.9\left(5 \times \mathrm{CH}_{2}, \mathrm{C}-\right.$
18-C-21, C-29), $27.18\left(\mathrm{CH}_{2}, \mathrm{C}-22\right)^{* 3}, 27.12\left(\mathrm{CH}_{2}, \mathrm{C}-\right.$ $28)^{*}, 25.8\left(\mathrm{CH}_{2}, \mathrm{C}-29\right), 25.6\left(\mathrm{CH}_{2}, \mathrm{C}-25\right), 24.6\left(\mathrm{CH}_{2}, \mathrm{C}-\right.$ 17), $23.5\left(\mathrm{CH}_{3}, \mathrm{C}-32\right), 22.1\left(\mathrm{CH}_{3}, \mathrm{C}-9\right), 21.2\left(\mathrm{CH}_{3}, \mathrm{C}-14\right)$, $19.8\left(\mathrm{CH}_{3}, \mathrm{C}-12\right), 14.1\left(\mathrm{CH}_{3}, \mathrm{C}-32\right), *^{1}, *^{2}, *^{3}$ assignments might be interchanged; ESIMS $\mathrm{m} / z$ 571.32 $[\mathrm{M}+\mathrm{H}]^{+}$, $569.30[\mathrm{M}-\mathrm{H}]^{-}$; HRESIMS $\mathrm{m} / \mathrm{z}, 571.3264[\mathrm{M}+\mathrm{H}]^{+}$ (calcd. for $\mathrm{C}_{33} \mathrm{H}_{47} \mathrm{O}_{8}, 571.3265$ ); $\mathrm{R}_{\mathrm{t}}=14.4$ min.

Fragirubrin E (7): Yellow oil; $[\alpha]_{\mathrm{D}}^{25}=+124^{\circ}(c=1.0$ $\left.\mathrm{CH}_{3} \mathrm{CN}\right)$; UV $\left(\mathrm{CH}_{3} \mathrm{CN}\right) \lambda_{\max }(\log \varepsilon): 218$ (4.08), $326 \mathrm{~nm}$ (4.00); $\mathrm{CD}\left(\mathrm{CH}_{3} \mathrm{CN}\right) \lambda(\Delta \varepsilon): 198(-4.9), 231(+1.6), 249$ $(-1.1), 273(+3.9), 323(-4.1) \mathrm{nm} ;{ }^{1} \mathrm{H}$ NMR $(700 \mathrm{MHz}$, $\left.\mathrm{CHCl}_{3}-d\right): \delta_{\mathrm{H}} 7.85(\mathrm{br} \mathrm{s}, 1-\mathrm{H}), 6.14(\mathrm{~s}, 4-\mathrm{H}), 5.54(\mathrm{br} \mathrm{s}, 5-$ H), 5.36-5.43 (m, 23-H, 26-H, 27-H), $5.33(\mathrm{~m}, 24-\mathrm{H})$, $5.17(\mathrm{dqd}, J=7.4,6.5,5.3 \mathrm{~Hz}, 11-\mathrm{H}), 3.56(\mathrm{~m}, 30-\mathrm{H})$, $2.80\left(\mathrm{t}, J=6.7 \mathrm{~Hz}, 25-\mathrm{H}_{2}\right), 2.69(\mathrm{dd}, J=14.8,7.5 \mathrm{~Hz}, 10$ $\left.\mathrm{H}_{\mathrm{a}}\right), \quad 2.61\left(\mathrm{dd}, \quad J=14.8, \quad 5.4 \mathrm{~Hz}, \quad 10-\mathrm{H}_{\mathrm{b}}\right), 2.45 \quad(\mathrm{t}$, $\left.J=7.5 \mathrm{~Hz}, 16-\mathrm{H}_{2}\right), 2.21\left(\mathrm{~m}, 28-\mathrm{H}_{\mathrm{a}}\right), 2.17\left(\mathrm{~m}, 28-\mathrm{H}_{\mathrm{b}}\right)$, $2.06\left(\mathrm{~s}, 14-\mathrm{H}_{3}\right), 2.04\left(\mathrm{~s}, 22-\mathrm{H}_{2}\right), 1.63\left(\mathrm{~m}, 17-\mathrm{H}_{2}\right), 1.55(\mathrm{~m}$, $\left.29-\mathrm{H}_{\mathrm{a}}\right), 1.53\left(\mathrm{~s}, 9-\mathrm{H}_{3}\right), 1.52\left(\mathrm{~m}, 31-\mathrm{H}_{\mathrm{a}}\right), 1.49\left(\mathrm{~m}, 29-\mathrm{H}_{\mathrm{b}}\right)$, $1.46\left(\mathrm{~m}, 31-\mathrm{H}_{\mathrm{b}}\right), 1.41\left(\mathrm{~m}, 29-\mathrm{H}_{\mathrm{b}}\right), 1.33(\mathrm{~d}, J=6.2 \mathrm{~Hz}$, $\left.12-\mathrm{H}_{3}\right), 1.25-1.38\left(\mathrm{~m}, 18-\mathrm{H}_{2}-21-\mathrm{H}_{2}\right), 0.95(\mathrm{t}, J=7.4 \mathrm{~Hz}$, $\left.32-\mathrm{H}_{3}\right) ;{ }^{13} \mathrm{C}$ NMR $\left(175 \mathrm{MHz}, \mathrm{CHCl}_{3}-d\right): \delta_{\mathrm{C}} 193.2(\mathrm{C}, \mathrm{C}-$ 8), 192.8 (C, C-6), 173.1 (C, C-15), 170.3 (C, C-13), 157.8 (C, C-3), 153.7 (CH, C-1), 142.0 (C, C-4a), 130.3 (CH, CH-23), 129.5 (CH, C-27), 128.6 (C, CH-26), 127.7 (CH, C-24), 115.2 (C, C-8a), 110.8 (CH, C-4), 107.5 (CH, $\mathrm{C}-5), 84.1$ (C, C-7), $72.9(\mathrm{CH}, \mathrm{C}-30), 67.5(\mathrm{CH}, \mathrm{C}-11)$, $39.4\left(\mathrm{CH}_{2}, \mathrm{C}-10\right), 36.7\left(\mathrm{CH}_{2}, \mathrm{C}-29\right), 33.2\left(\mathrm{CH}_{2}, \mathrm{C}-16\right)$, $30.2\left(\mathrm{CH}_{2}, \mathrm{C}-31\right), 29.5\left(\mathrm{CH}_{2}, \mathrm{C}-21\right), 29.1,29.0\left(2 \times \mathrm{CH}_{2}\right.$, $\mathrm{C}-19, \mathrm{C}-20), 28.9\left(\mathrm{CH}_{2}, \mathrm{C}-18\right), 27.2\left(\mathrm{CH}_{2}, \mathrm{C}-22\right), 25.6$ $\left(\mathrm{CH}_{2}, \mathrm{C}-25\right), 24.6\left(\mathrm{CH}_{2}, \mathrm{C}-17\right), 23.6\left(\mathrm{CH}_{3}, \mathrm{C}-28\right), 22.1$ $\left(\mathrm{CH}_{3}, \mathrm{C}-9\right), 21.2\left(\mathrm{CH}_{3}, \mathrm{C}-14\right), 19.8\left(\mathrm{CH}_{3}, \mathrm{C}-12\right), 9.9$ $\left(\mathrm{CH}_{3}, \mathrm{C}-32\right)$; ESIMS $\mathrm{m} / z \quad 571.32[\mathrm{M}+\mathrm{H}]^{+}, \quad 569.30$ $[\mathrm{M}-\mathrm{H}]^{-}$; HRESIMS $m / z 571.3260[\mathrm{M}+\mathrm{H}]^{+}$(calcd. for $\left.\mathrm{C}_{33} \mathrm{H}_{47} \mathrm{O}_{8}, 571.3265\right) ; \mathrm{R}_{\mathrm{t}}=14.5$ min.

Lenormandin F (8): Yellow oil; UV $\left(\mathrm{CH}_{3} \mathrm{CN}\right) \lambda_{\max }(\log$ ع): $222,328 \mathrm{~nm} ; \mathrm{CD}\left(\mathrm{CH}_{3} \mathrm{CN}\right) \lambda(\Delta \varepsilon): 199(-6.5), 234$ $(+2.8), 250(-1.0), 273(+4.8), 323(-4.1) \mathrm{nm} ;{ }^{1} \mathrm{H}$ NMR (500 MHz, $\left.\mathrm{CHCl}_{3}-d\right): \delta_{\mathrm{H}} 7.88(\mathrm{br} \mathrm{s}, 1-\mathrm{H}), 6.20(\mathrm{~s}, 4-$ H), 5.55 (br s, 5-H), 4.17 (dqd, $J=7.4,6.5,5.3 \mathrm{~Hz}, 11-\mathrm{H})$, $2.54\left(\mathrm{~m}, 10-\mathrm{H}_{2}\right), 2.44\left(\mathrm{t}, J=7.5 \mathrm{~Hz}, 14-\mathrm{H}_{2}\right), 1.63(\mathrm{~m}, 15-$ $\left.\mathrm{H}_{2}\right), 1.53\left(\mathrm{~s}, 9-\mathrm{H}_{3}\right), 1.32\left(\mathrm{~d}, J=6.5 \mathrm{~Hz}, 12-\mathrm{H}_{3}\right), 1.23-1.35$ $\left(\mathrm{m}, 16-\mathrm{H}_{2}-27-\mathrm{H}_{2}\right), 0.89\left(\mathrm{t}, J=6.9 \mathrm{~Hz}, 28-\mathrm{H}_{3}\right) ;{ }^{13} \mathrm{C} \mathrm{NMR}$ $\left(125 \mathrm{MHz}, \mathrm{CHCl}_{3}-d\right): \delta_{\mathrm{C}} 193.4(\mathrm{C}, \mathrm{C}-8), 192.9(\mathrm{C}, \mathrm{C}-6)$, 173.1 (C, C-13), 159.0 (C, C-3), 153.8 (CH, C-1), 142.2 (C, C-4a), $115.2(\mathrm{C}, \mathrm{C}-8 \mathrm{a}), 110.6(\mathrm{CH}, \mathrm{C}-4), 107.3(\mathrm{CH}$, $\mathrm{C}-5), 84.0(\mathrm{C}, \mathrm{C}-7), 65.4(\mathrm{CH}, \mathrm{C}-11), 42.5\left(\mathrm{CH}_{2}, \mathrm{C}-10\right)$, $33.2\left(\mathrm{CH}_{2}, \mathrm{C}-14\right), 31.9\left(\mathrm{CH}_{2}, \mathrm{C}-26\right), 29.68,29.67,29.66$, 29.64, 29.63, 29.61, 29.41, 29.35, $29.26\left(9 \times \mathrm{CH}_{2}, \mathrm{C}-17-\right.$ $\mathrm{C}-25), 28.9\left(\mathrm{CH}_{2}, \mathrm{C}-16\right), 24.6\left(\mathrm{CH}_{2}, \mathrm{C}-15\right), 23.6\left(\mathrm{CH}_{3}, \mathrm{C}-\right.$ 12), $22.7\left(\mathrm{CH}_{2}, \mathrm{C}-27\right), 22.1\left(\mathrm{CH}_{3}, \mathrm{C}-9\right), 14.1\left(\mathrm{CH}_{3}, \mathrm{C}-28\right)$; ESIMS $m / z \quad 489.32[\mathrm{M}+\mathrm{H}]^{+}, \quad 487.32 \quad[\mathrm{M}-\mathrm{H}]^{-}$; 
HRESIMS $m / z 489.3217[\mathrm{M}+\mathrm{H}]^{+}$(calcd. for $\mathrm{C}_{29} \mathrm{H}_{45} \mathrm{O}_{6}$, 489.3211); $\mathrm{R}_{\mathrm{t}}=17.8 \mathrm{~min}$; data are in good agreement with the literature (Kuhnert et al. 2014c).

\section{Mosheŕs analyses}

For the preparation of the (S)-MTPA ester $0.2 \mathrm{mg}$ of $\mathbf{8}$ was dissolved in $600 \mu \mathrm{l}$ of pyridine- $d_{5}$, and $10 \mu \mathrm{l}$ of $(R)$-MTPA chloride was added. The mixture was incubated at $25{ }^{\circ} \mathrm{C}$ for 15 min before measurement of ${ }^{1} \mathrm{H}, \mathrm{COSY}$, TOCSY and HSQC NMR spectra: ${ }^{1} \mathrm{H}$ NMR $\left(700 \mathrm{MHz}\right.$, pyridine- $\left.d_{5}\right): \delta_{\mathrm{H}}$ $8.22(1-\mathrm{H}), 6.32(4-\mathrm{H}), 5.76(5-\mathrm{H}), 5.55(11-\mathrm{H}), 2.79$ (10$\left.\mathrm{H}_{2}\right), 1.73\left(9-\mathrm{H}_{3}\right), 1.33\left(7-\mathrm{H}_{3}\right)$. The $(R)$-MTPA ester was prepared in the same manner by the addition of $10 \mu \mathrm{L}$ of $(S)$-MTPA chloride: ${ }^{1} \mathrm{H}$ NMR $\left(700 \mathrm{MHz}\right.$, pyridine- $\left.d_{5}\right): \delta_{\mathrm{H}}$ $8.13(1-\mathrm{H}), 6.04(4-\mathrm{H}), 5.63(5-\mathrm{H}), 5.56(11-\mathrm{H}), 2.72(10-$ $\left.\mathrm{H}_{2}\right), 1.72\left(9-\mathrm{H}_{3}\right), 1.38\left(12-\mathrm{H}_{3}\right)$.

Fragirubrin D $(2 \times 0.2 \mathrm{mg}, 6)$ was converted analogously. (S)-MTPA ester of 6: ${ }^{1} \mathrm{H}$ NMR $(700 \mathrm{MHz}$, pyridine- $\left.d_{5}\right)$ : similar to 6 , but $\delta_{\mathrm{H}} 5.29(31-\mathrm{H}), 1.63\left(30-\mathrm{H}_{\mathrm{a}}\right)$, $1.53\left(30-\mathrm{H}_{\mathrm{b}}\right), 1.31\left(32-\mathrm{H}_{3}\right) ;(R)$-MTPA ester of $6:{ }^{1} \mathrm{H}$ NMR (700 MHz, pyridine- $\left.d_{5}\right)$ : similar to 6 , but $\delta_{\mathrm{H}} 5.28(31-\mathrm{H})$, $1.70\left(30-\mathrm{H}_{\mathrm{a}}\right), 1.58\left(30-\mathrm{H}_{\mathrm{b}}\right), 1.24\left(32-\mathrm{H}_{3}\right)$.

Fragirubrin E $(2 \times 0.2 \mathrm{mg}, 7)$ was converted analogously. (S)-MTPA ester of 7: ${ }^{1} \mathrm{H}$ NMR $(700 \mathrm{MHz}$, pyridine- $\left.d_{5}\right)$ : similar to 7 , but $\delta_{\mathrm{H}} 5.22(30-\mathrm{H}), 2.13\left(28-\mathrm{H}_{\mathrm{a}}\right)$, $2.05\left(28-\mathrm{H}_{\mathrm{b}}\right), 1.71\left(29-\mathrm{H}_{\mathrm{a}}\right), 1.65\left(29-\mathrm{H}_{\mathrm{b}}\right), 1.68\left(31-\mathrm{H}_{2}\right)$, $0.90\left(32-\mathrm{H}_{3}\right) ;(R)$-MTPA ester of 7: ${ }^{1} \mathrm{H}$ NMR $(700 \mathrm{MHz}$, pyridine- $\left.d_{5}\right)$ : similar to 7 , but $\delta_{\mathrm{H}} 5.23(30-\mathrm{H}), 2.26\left(28-\mathrm{H}_{\mathrm{a}}\right)$, $2.22\left(28-\mathrm{H}_{\mathrm{b}}\right), 1.81\left(29-\mathrm{H}_{\mathrm{a}}\right), 1.70\left(29-\mathrm{H}_{\mathrm{b}}\right), 1.61\left(31-\mathrm{H}_{2}\right)$, $0.79\left(32-\mathrm{H}_{3}\right)$.

\section{Results}

Over 100 fragments that were reminiscent of xylarialean stromata were studied microscopically, but most of those did not contain any material that would allow for their concise identification. Seven samples that contained more or less intact ascospores that allowed for determination of their dimensions and shape were submitted concurrently to high performance chromatography coupled with diode array and mass spectrometric detection (HPLC-DAD/MS) and also studied using scanning electron microscopy.

Four of these samples were identified as Hypoxylon fragiforme, two were assigned to Jackrogersella cohaerens and one was identified tentatively as Hypoxylon cf. vogesiacum. The Jackrogersella cohaerens specimens were identified by their characteristic stromatal and ascospore morphology and also contained traces of the characteristic pigments, cohaerins A and B (Quang et al. 2005b). Other cohaerins, such as the compounds described by Quang et al. (2006) and Surup et al. (2013) were not detected.
The specimen of $H$. cf. vogesiacum was determined by morphology only, since the HPLC profiles were inconclusive. However, the rather large ascospores strongly pointed toward the identity of this species, and at least from Europe, no other species except for the artic-alpine $H$. macrosporum has ascospores of similar size. The latter species also features olivaceous stromatal pigments, due to the presence of daldinin type azaphilones (Stadler et al. 2008b), whereas the stroma we studied did not give any pigment reaction, as is often the case even in freshly collected $H$. vogesicacum.

The $H$. fragiforme specimens all contained the typical metabolite patterns, including orsellinic acid (9) and the azaphilones, mitorubrin (10), mitorubrinol (11), and mitorubrinol acetate (12) as major metabolites. Furthermore, the extracts contained many peaks corresponding to hitherto unknown metabolites ( $c f$ Fig. 4), which could not be assigned to any known metabolites of the Xylariales according to our database search. Since these metabolites were also encountered in fresh material, large quantities of stromata were collected in the field. Subsequently, the isolation of sufficient amounts allowed for the structure elucidation of the novel metabolites 1-7. Compound 8 was found to be lenormandin F (Kuhnert et al. 2014c).

Rutilin C (1) was isolated as a red oil; its molecular ion cluster in the HRESIMS spectrum revealed the molecular formula $\mathrm{C}_{42} \mathrm{H}_{34} \mathrm{O}_{14}$. The proton and ${ }^{1} \mathrm{H},{ }^{13} \mathrm{C}$ HSQC NMR spectra of $\mathbf{1}$ showed the presence of five methyls, one methylene and 13 olefinic/aromatic methines. The ${ }^{13} \mathrm{C}$ and ${ }^{1} \mathrm{H},{ }^{13} \mathrm{C}$ HMBC spectra furthermore indicated four conjugated ketones, two carboxyls, and $15 \mathrm{sp}^{2}$ hybridized plus two $\mathrm{sp}^{3}$ hybridized further carbons without bound protons. By ${ }^{1} \mathrm{H},{ }^{13} \mathrm{C}$ HMBC correlations, two orsellinic acid and two azaphilone subunits were identified. COSY and TOCSY correlations connected methines $12 \mathrm{a}-\mathrm{H} / 13 \mathrm{a}-\mathrm{H} / 14 \mathrm{a}-\mathrm{H}$ and a propenyl side chain comprising $12 \mathrm{~b}-\mathrm{H} / 13 \mathrm{~b}-\mathrm{H} / 14 \mathrm{~b}-\mathrm{H}_{3}$, which were linked to $\mathrm{C}-3 \mathrm{a}$ and $\mathrm{C}-3 \mathrm{~b}$ by $\mathrm{HMBC}$ correlations, respectively. $\mathrm{HMBC}$ correlations from $14 \mathrm{a}-\mathrm{H}$ to $\mathrm{C}-7 \mathrm{~b} / \mathrm{C}-6 \mathrm{~b} / \mathrm{C}-5 \mathrm{~b}$ and $4 \mathrm{~b}-\mathrm{H}$ to $\mathrm{C}-5 \mathrm{~b} / \mathrm{C} 6 \mathrm{~b}$ revealed the planar core structure. The $\mathrm{CD}$ spectrum of $\mathbf{1}$ with its negative cotton effect at $365 \mathrm{~nm}$ and positive effects at 260 and $450 \mathrm{~nm}$ confirmed the $8 \mathrm{a} R / 8 \mathrm{~b} R$ stereochemistry alike the biosynthetically related mitorubrins (Clark et al. 2008). Taken together, the compound constitutes the 7a-dehydro14b-desacetoxy derivative of rutilin $\mathrm{B}$, which is known from Hypoxylon rutilum. Due to the structural similarity to rutilin $\mathrm{B}$, we chose to name $\mathbf{1}$ as rutilin $\mathrm{C}$.

The red oil rutilin D (2) was analyzed for a molecular formula $\mathrm{C}_{44} \mathrm{H}_{36} \mathrm{O}_{16}$, indicating the formal addition of a $\mathrm{C}_{2} \mathrm{H}_{2} \mathrm{O}_{2}$ fragment in comparison to 1 . The NMR spectra of $\mathbf{2}$ were highly similar to those of $\mathbf{1}$. The key difference was the replacement of methyl $14 \mathrm{~b}$ by an oxymethylene, and signals for an additional acetyl moiety, which was 


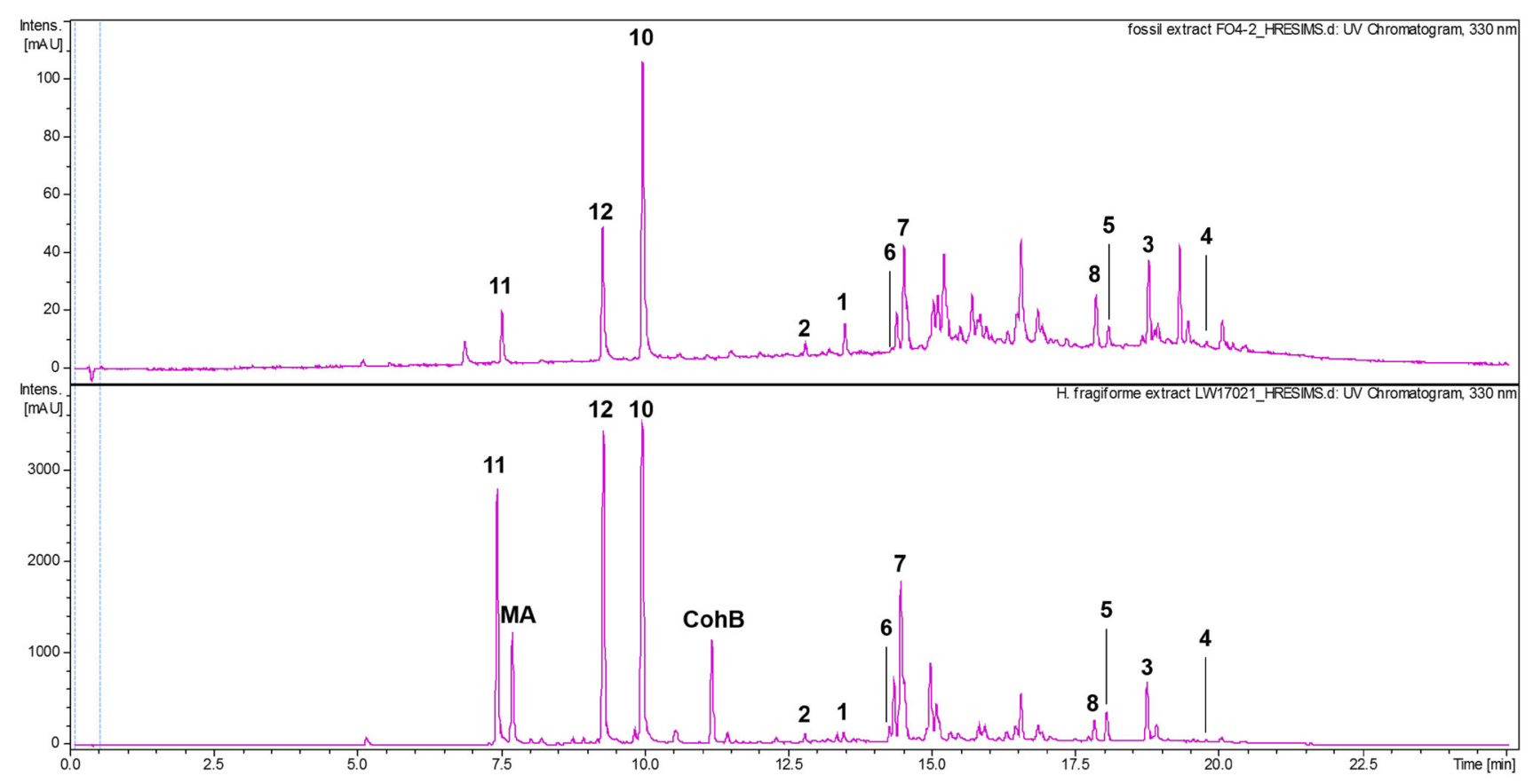

Fig. 4 HPLC-UV/VIS chromatogram (at $330 \mathrm{~nm}$ ) of the fossil sample Fo4-2, depicted in Fig. 1 (upper part) and the large scale extraction from fresh stromatal material STMA18022 (lower part).

connected to $14 \mathrm{~b}$ due to the HMBC correlation from $14 \mathrm{~b}-\mathrm{H}$ to $\mathrm{C}$-15. Therefore, 2 was identified as the $14 \mathrm{~b}$-acetoxy derivative of $\mathbf{1}$ and named rutilin D (Fig. 5).

Fragirubrin A (3) was isolated as yellow oil. Its HRESIMS data indicated the molecular formula $\mathrm{C}_{31} \mathrm{H}_{46} \mathrm{O}_{7}$, and thereby nine degrees of unsaturation. The ${ }^{1} \mathrm{H}$ and ${ }^{1} \mathrm{H},{ }^{13} \mathrm{C}$ HSQC NMR spectra of 3 showed four methyls, three olefinic and one aliphatic methines and 15 methylenes. In addition, the ${ }^{13} \mathrm{C}$ spectrum provided evidence for two conjugated ketones, two ester carbonyls, one oxygenated $\mathrm{sp}^{3}$ hybridized and three $\mathrm{sp}^{2}$ hybridized carbons devoid of bound protons. ${ }^{1} \mathrm{H},{ }^{13} \mathrm{C} \mathrm{HMBC}$ correlations from 1-H to C-3, C-4a, C-8, C-8a; from 4-H to C-3, C-4a, C5, C-8a; from 5-H to C-4, C-4a, C-7, C-8a; and from 9$\mathrm{H}_{3}$ to $\mathrm{C}-6, \mathrm{C}-7, \mathrm{C}-8$ identified a highly conjugated azaphilone core structure. COSY correlations between $10-\mathrm{H}_{2}$, 11- $\mathrm{H}$ and 12- $\mathrm{H}_{3}$ assigned a 2-hydroxypropyl moiety, attached to $\mathrm{C}-3$ due to $\mathrm{HMBC}$ correlations from $10-\mathrm{H}_{\mathrm{a} / \mathrm{b}}$ to $\mathrm{C}-3, \mathrm{C}-4$ and $\mathrm{H}-4$ to $\mathrm{C}-10$. Another entity was assigned as palmitinyl based on COSY and TOCSY correlations from both $17-\mathrm{H}_{2} / 18-\mathrm{H}_{2}$ and $19-\mathrm{H}_{2} / 30-\mathrm{H}_{2}$ to the unresolved $\mathrm{CH}_{2}$ region. The fatty acid side chain was connected to $\mathrm{C}-7$ based on the missing valences, finalizing the planar structure of $\mathbf{3}$. The stereochemistry of C-7 was addressed by CD spectroscopy. The CD spectrum of $\mathbf{3}$ showed a negative cotton effect at 347 and positive effects at 273 , indicating a $7 R$ stereochemistry alike to the structurally closely related mitorubrins (Clark et al. 2008). The absolute
Orsellinic acid (9) cannot be observed at $330 \mathrm{~nm}$ but elutes after $\mathrm{RT}=3.63 \mathrm{~min}$. Numbers correspond to compound identifiers used in this article. $\boldsymbol{M A}$ mitorubrinic acid, $\boldsymbol{C o h B}$ cohaerin B

stereochemistry of C-11 was assigned by Mosher's method (Hoye et al. 2007), utilizing 8, the deacetyl-derivative of $\mathbf{3}$. Negative $\Delta \delta^{\mathrm{SR}}$ values of $11-\mathrm{H}$ and $12-\mathrm{H}_{3}$ and positive ones for all other protons indicated a $11 S$ configuration of the metabolite family; the same stereochemistry had been determined for lenormandin $\mathrm{F}$ isolated from $H$. lenormandii (Kuhnert et al. 2014c).

Fragirubrin $\mathrm{B}(4)$ had the molecular formula $\mathrm{C}_{33} \mathrm{H}_{50} \mathrm{O}_{7}$, indicating the formal addition of a $\mathrm{C}_{2} \mathrm{H}_{4}$ fragment compared to 3. Since the NMR spectra of $\mathbf{4}$ were virtually identical to those of $\mathbf{3}$, the presence of a stearyl fatty acid side chain was deduced.

For fragirubrin $\mathrm{C} \mathrm{(5)} \mathrm{a} \mathrm{molecular} \mathrm{formula} \mathrm{C}_{33} \mathrm{H}_{46} \mathrm{O}_{7}$ was identified by HRESIMS data, indicating two additional degrees of unsaturation compared to 3 . The proton spectrum of $\mathbf{5}$ was similar to that of $\mathbf{3}$, with the exception of four additional olefinic methines between $\delta_{\mathrm{H}}$ 5.30-5.40. The chemical shift of $25-\mathrm{H}_{2}\left(\delta_{\mathrm{H}} 2.77\right)$ indicated that this methylene is surrounded by two double bonds. The double bond geometry was deduced as $Z$ for both ones, since ROESY correlations were observed between $25-\mathrm{H}_{2}$ and $22-\mathrm{H}_{2} / 28-\mathrm{H}_{2}$, but not between $22-\mathrm{H}_{2} / 28-\mathrm{H}_{2}$ and $24-\mathrm{H} /$ $26-\mathrm{H}$. Due to $\mathrm{HMBC}$ correlations from $28-\mathrm{H}_{2}$ to $29-\mathrm{H}_{2} /$ $30-\mathrm{H}_{2}$ and $32-\mathrm{H}_{3}$ to $30-\mathrm{H}_{2} / 31-\mathrm{H}_{2}$, the fatty acid side chain was identified as linoleic acid.

The molecular formula of fragirubrin $\mathrm{D}(\mathbf{6})$ was determined as $\mathrm{C}_{33} \mathrm{H}_{46} \mathrm{O}_{8}$, indicating an additional oxygen atom compared to 5. The key difference between the highly 
<smiles>[R]O[C@]1(C)C(=O)C=C2C=C(C[C@H](C)OC(C)=O)OC=C2C1=O</smiles><smiles></smiles><smiles>[R][R]=P[R](C)(C)OC(C)=O</smiles><smiles></smiles><smiles>[R]C/C=C/C1=CC2=C(CO1)C(=O)[C@](C)(O)C(=O)C2=CC</smiles>

5: $\mathrm{R}=$<smiles>CCCCC/C=C\C/C=C\CCCCCCCC(=O)C(C)(C)C</smiles>

6: $\mathrm{R}$<smiles>[R]=[Fe]C(=O)CCCCCCC/C=C\C/C=C\CCC[C@H](C)O</smiles>

7: $\mathrm{R}=$<smiles>CC[C@H](O)CC/C=C\C/C=C\CCCCCCCC(C)=O</smiles><smiles>C[C@@H](O)CC1=CC2=CC(=O)[C@](C)(O)C(=O)C2=CO1</smiles><smiles>O=C([O-])c1[13cH][13cH][13c](O)[13c]c1O</smiles><smiles>O=C(O)CO</smiles>

8

10: $R^{2}=H$

11: $\mathrm{R}^{2}=\mathrm{OH}$

12: $\mathrm{R}^{2}=\mathrm{C}_{\mathrm{O}}$

Fig. 5 Stromatal metabolites detected in the current study. 1: rutilin C; 2: rutilin D; 3: fragirubrin A; 4: fragirubrin B; 5: fragirubrin C; 6: fragirubrin D; 7: fragirubrin E; 8: lenormandin F; 9: orsellinic acid; 10: mitorubrin; 11: mitorubrinol; 12: mitorubrinol acetate

similar ${ }^{1} \mathrm{H}$ and ${ }^{13} \mathrm{C}$ NMR spectra of $\mathbf{6}$ and 5 was the replacement of a methylene by an oxymethine. Since the additional oxymethine $31-\mathrm{H}$ showed a COSY correlation to $32-\mathrm{H}_{3}$, whose triplet of $\mathbf{3}$ was replaced by a doublet in $\mathbf{6}$, $\mathbf{6}$ was identified as the 31-hydroxy derivative of $\mathbf{5}$. The absolute stereochemistry of C-31 was assigned by Mosher's method. Negative $\Delta \delta^{\mathrm{SR}}$ values of $30-\mathrm{H}_{\mathrm{a}}$ and $30-\mathrm{H}_{\mathrm{b}}$ and a positive one for $32-\mathrm{H}_{3}$ indicated a $31 R$ configuration.

As shown by its HRESIMS data, fragirubrin E (7) has the same molecular formula $\mathrm{C}_{33} \mathrm{H}_{46} \mathrm{O}_{8}$ as 6 . The NMR spectra of 7 were highly similar to those of $\mathbf{6}$, with the major difference of methyl $32-\mathrm{H}_{3}$ showing a triplet instead of a dublet. Due to the HMBC correlation of $32-\mathrm{H}_{3}$ to oxymethine $30-\mathrm{H}, 7$ was identified as the 30-hydroxy derivative of $\mathbf{5}$. The absolute stereochemistry of $\mathrm{C}-30$ was again assigned by Mosher's method. Negative $\Delta \delta^{\mathrm{SR}}$ values of $28-\mathrm{H}_{\mathrm{a}}, 28-\mathrm{H}_{\mathrm{b}} 29-\mathrm{H}_{\mathrm{a}}$ and $30-\mathrm{H}_{\mathrm{b}}$ and positive ones for $31-\mathrm{H}_{2}$ and $32-\mathrm{H}_{3}$ indicated a $30 R$ configuration.

\section{Discussion}

The current study is to the best of our knowledge the first one where secondary metabolites from fungi have been detected in ancient materials that are estimated to be many centuries old. It was already surprising to see that the specimens still contained ascospores, and this may be due to the fact that the Xylariales often have the ability to form rather persistent spores. However, the spores were not apparently intact (e.g., the perispore could not be seen) and hence, their examination and the macroscopic characters of the stromata still left some doubt as to the identity of the material and only the fact that the secondary metabolites were rather well preserved allowed for a safe identification of the species.

Some metabolites involved were already known to be relatively stable and had repeatedly been detected in herbarium specimens that had been kept in museums. For instance, mitorubrins and the related rubiginosins have been detected in various specimens of Hypoxylon by 
Stadler et al. (2008a) and Entonaema (Stadler et al. 2008b) that were collected during the nineteenth century.

The present study reports on the occurrence of conjugated bisazaphilones in $H$. fragiforme for the first time. However, structurally related compounds have already been obtained previously from different species of the Hypoxylaceae, including the rutilins from $H$. rutilum (Quang et al. 2005a) and entonaemin C from a fungus that was referred to as Entonaema splendens collected in Japan (Hashimoto and Asakawa 1998). The identification of the latter species was probably not correct, since E. splendens is a name referring to a Caribbean species whose type features immature stromata, but similar compounds were detected by HPLC-DAD/MS in both, E. cinnabarinum and E. liquescens (Stadler et al. 2008b). It is difficult to tell why these compounds have persisted in the stromata for such a long time because in particular the azaphilones are regarded to be rather reactive, and the trivial name of this metabolite class even relates to their ability to spontaneously incorporate nitrogen (Gao et al. 2013). On the other hand, as shown by the illustrations provided by Stadler and Fournier (2006) and Stadler et al. (2006), they are contained in the mature stromata in rather large amounts and are embedded in a waxy layer beneath the stromata surface, surrounding the perithecia, where they are normally not exposed to the environment. This feature seems to have determined the longevity of the secondary metabolites of many type and historical specimens and now it has even been proven that the metabolites remain stable for up to a millenium. We had been hoping to find evidence, e.g. for the presence of new taxa, or of tropical fungi that may have dominated the forests of Central France during medieval times, but according to the evidence provided in this study, we have mostly found the same fungi that still dominate the beech forests of the temperate Northern hemisphere. However, our studies are going on, and we have not yet studied many of the fragments that resulted from the excavation.

We are not aware of any previous study on the presence of secondary metabolites in ancient fungal specimens, and do not think this could be a general option to establish in palaeontology. After all, most of the petrified fossil fungal specimens that have been dealt with previously are not very likely to yield conclusive data on secondary metabolites, even if the most sensitive methods of analytical chemistry will be employed. Due to the fact that we only observed highly carbonised stromata, deflated hyphae and no intact ascospores, even by SEM, we can exclude that it would still be possible to obtain DNA from the ancient material. It should be very interesting to see whether a similar approach will succeed with other samples that have been collected during excavations that are intended to study the recent human history.
Our methodology is well-suited to study further fungal specimens including those that have been dated and proven to be much older than the current material as outlined by Knoll (2014), palaeobiology does already rely on microscopic studies including SEM, but the reconstruction of the early evolution of Eukaryotes is hampered by the lack of data on fossils. In particular, the newly arising Molecular Clock studies (Divakar et al. 2017; Hongsanan et al. 2017) that rely on temporal phylogenies would certainly work better if more fossils with reliably determined age were available. The future will show whether chemotaxonomic traits can be employed in such endeavours.

Acknowledgements We wish to thank Prof. D. L. Hawksworth for establishing contact between the working groups in France and Germany. AN is indebted for a grant of the Iranian government for a research stay in Germany. LW is grateful for a $\mathrm{PhD}$ grant from the province government of Lower Saxony (HSBDR graduate school). KB and MS are grateful for a grant from the Deutsche Forschungsgemeinschaft (DFG) in the Priority Programme "Taxon-Omics: New Approaches for Discovering and Naming Biodiversity" (SPP 1991). Christel Kakoschke, Cäcilia Schwager, Aileen Gollasch, Anke Skiba and Vanessa Stiller are thanked for expert technical assistance. We are grateful to Annelise Binois for her helpful comments on the manuscript.

\section{References}

Bitzer J, Köpcke B, Stadler M, Hellwig V, Ju YM, Seip S, Henkel T (2007) Accelerated dereplication of natural products, supported by reference libraries. Chimia 51:332-338

Chapela IH, Petrini O, Bielser G (1993) The physiology of ascospore eclosion in Hypoxylon fragiforme: mechanisms in the early recognition and establishment of an endophytic symbiosis. Mycol Res 97:157-162

Clark RC, Lee SY, Boger DL (2008) Total synthesis of chlorofusin, its seven chromophore diastereomers, and key partial structures. J Am Chem Soc 130:12355-12369

Daranagama DA, Hyde KD, Sir EB, Thambugala KM, Tian Q, Samarakoon MC, McKenzie EHC, Jayasiri SC, Tibpromma S, Bhat JD, Liu X, Stadler M (2018) Towards a natural classification and backbone tree for Graphostromataceae, Hypoxylaceae, Lopadostomataceae and Xylariaceae. Fungal Divers 88:1-165

Divakar PK, Crespo A, Kraichak E, Leavitt SD, Singh G, Schmitt I, Lumbsch HT (2017) Using a temporal phylogenetic method to harmonize family-and genus-level classification in the largest clade of lichen-forming fungi. Fungal Divers 84:101-117

Fournier J, Köpcke B, Stadler M (2010) New species of Hypoxylon from western Europe and Ethiopia. Mycotaxon 113:209-235

Gao J-M, Yang S-X, Qin J-C (2013) Azaphilones: chemistry and Biology. Chem Rev 113:4755-4811

Hashimoto T, Asakawa Y (1998) Biologically active substances of Japanese inedible mushrooms. Heterocycles 2(47):1067-1110

Helaly SE, Thongbai B, Stadler M (2018) Diversity of biologically active secondary metabolites from endophytic and saprotrophic fungi of the ascomycete order Xylariales. Nat Prod Rep. https:// doi.org/10.1039/c8np00010g

Hellwig V, Ju Y-M, Rogers JD, Fournier J, Stadler M (2005) Hypomiltin, a novel azaphilone from Hypoxylon hypomiltum, 
and chemotypes in Hypoxylon sect. Hypoxylon as inferred from analytical HPLC profiling. Mycol Progr 4:39-54

Hongsanan S, Maharachchikumbura SS, Hyde KD, Samarakoon MC, Jeewon R, Zhao Q, Al-Sadi AM, Bahkali AH (2017) An updated phylogeny of Sordariomycetes based on phylogenetic and molecular clock evidence. Fungal Divers 84:25-41

Hoye TR, Jeffrey CS, Shao F (2007) Mosher ester analysis for the determination of absolute configuration of stereogenic (chiral) carbinol carbons. Nat Protoc 2:2451-2458

Knoll AH (2014) Paleobiological perspectives on early eukaryotic evolution. Cold Spring Harbor Perspect Biol 6(1):a016121

Kuhnert E, Fournier J, Peršoh D, Luangsa-ard JJ, Stadler M (2014a) New Hypoxylon species from Martinique and new evidence on the molecular phylogeny of Hypoxylon based on ITS rDNA and $\beta$-tubulin data. Fungal Divers 64:181-203

Kuhnert E, Heitkämper S, Fournier J, Surup F, Stadler M (2014b) Hypoxyvermelhotins A-C, new pigments from Hypoxylon lechatii sp. nov. Fungal Biol 118:242-252

Kuhnert E, Surup F, Sir EB, Lambert C, Hyde KD, Hladki AI, Romero AI, Stadler M (2014c) Lenormandins A-G, new azaphilones from Hypoxylon lenormandii and Hypoxylon jaklitschii sp. nov., recognised by chemotaxonimic data. Fungal Divers 71:165-184. https://doi.org/10.1007/s13225-014-0318-1

Kuhnert E, Surup F, Herrmann J, Huch V, Müller R, Stadler M (2015) Rickenyls A-E, antioxidative terphenyls from the fungus Hypoxylon rickii (Xylariaceae, Ascomycota). Phytochemistry 118:68-73

Kuhnert E, Sir EB, Lambert C, Hyde KD, Hladki AI, Romero AI, Rohde M, Stadler M (2017) Phylogenetic and chemotaxonomic resolution of the genus Annulohypoxylon (Xylariaceae) including four new species. Fungal Divers 85:1-43

Provost M (2009) Carte archéologique de la Gaule, 21, La Côte-d'Or. Vol. 3, De Nuits-Saint-Georges à Voulaines-les-Templiers. Paris, France : Académie des inscriptions et belles-lettres: Ministère de l'éducation nationale : Ministère de la recherche

Quang DN, Hashimoto T, Stadler M, Asakawa Y (2005a) Dimeric azaphilones from the xylariaceous ascomycete Hypoxylon rutilum. Tetrahedron 61:8451-8455

Quang DN, Hashimoto T, Nomura Y, Wollweber H, Hellwig V, Fournier J, Stadler M, Asakawa Y (2005b) Cohaerins A and B, azaphilones from the fungus Hypoxylon cohaerens, and comparison of HPLC-based metabolite profiles in Hypoxylon sect. Annulata. Phytochemistry 66:797-809

Quang DN, Stadler M, Fournier J, Tomita A, Hashimoto T (2006) Cohaerins $\mathrm{C}-\mathrm{F}$, four azaphilones from the xylariaceous fungus Annulohypoxylon cohaerens. Tetrahedron 62:6349-6354
Sir EB, Kuhnert E, Lambert C, Hladki AI, Romero AI, Stadler M (2016) New species and reports of Hypoxylon from Argentina recognized by a polyphasic approach. Mycol Progr 15:42

Stadler M (2011) Importance of secondary metabolites in the Xylariaceae as parameters for assessment of their taxonomy, phylogeny, and functional biodiversity. Curr Res Envion Appl Mycol 1:75-133

Stadler M, Fournier J (2006) Pigment chemistry, taxonomy and phylogeny of the Hypoxyloideae (Xylariaceae). Rev Iberoam Micol 23:160-170

Stadler M, Quang DN, Tomita A, Hashimoto T, Asakawa Y (2006) Production of bioactive metabolites during stromatal ontogeny of Hypoxylon fragiforme. Mycol Res 110:811-820

Stadler M, Fournier J, Beltrán-Tejera E, Granmo A (2008a) The "red Hypoxylons" of the temperate and subtropical Northern Hemisphere. In "A Festschrift in honor of Professor Jack D. Rogers (Glawe DA, Ammirati JF, eds.). N Am Fungi 3:73-125

Stadler M, Fournier J, Læssøe T, Lechat C, Tichy HV, Piepenbring M (2008b) Recognition of hypoxyloid and xylarioid Entonaema species from a comparison of holomorphic morphology, HPLC profiles, and ribosomal DNA sequences. Mycol Progr 7:53-73

Stadler M, Læssøe T, Fournier J, Decock C, Schmieschek B, Tichy HV, Peršoh D (2014) A polyphasic taxonomy of Daldinia (Xylariaceae). Stud Mycol 77:1-143

Surup F, Mohr KI, Jansen R, Stadler M (2013) Cohaerins G-K, azaphilone pigments from Annulohypoxylon cohaerens and absolute stereochemistry of cohaerins C-K. Phytochemistry 95:252-258

Surup F, Kuhnert E, Lehmann E, Heitkämper S, Hyde KD, Fournier J, Stadler M (2014) Sporothriolide derivatives as chemotaxonomic markers for Hypoxylon monticulosum. Mycol Int J Fungal Biol 5:110-119

Surup F, Kuhnert E, Böhm A, Pendzialek T, Solga D, Wiebach V, Engler H, Berkessel A, Stadler M, Kalesse M (2018) The rickiols, 20-, 22-, and 24-membered macrolides from the ascomycete Hypoxylon rickii. Chem Eur J 24:2200-2213

Wendt L, Sir EB, Kuhnert E, Heitkämper S, Lambert C, Hladki AI, Romero AI, Luangsa-ard JJ, Srikitikulchai P, Peršoh D, Stadler M (2018) Resurrection and emendation of the Hypoxylaceae, recognised from a multi-gene genealogy of the Xylariales. Mycol Prog 17:115-154 